Pré-Publicações do Departamento de Matemática

Universidade de Coimbra

Preprint Number 08-35

\title{
VARIATIONAL IMAGE SEGMENTATION FOR ENDOSCOPIC HUMAN COLONIC ABERRANT CRYPT FOCI
}

\author{
I. N. FIGUEIREDO, P. N. FIGUEIREDO, G. STADLER, O. GHATTAS AND A. ARAÚJO
}

\begin{abstract}
The aim of this paper is to introduce a variational image segmentation method for assessing the aberrant crypt foci (ACF) in the human colon captured in vivo by endoscopy. ACF are thought to be precursors for colorectal cancer, and therefore their early detection may play an important clinical role. We enhance the active contours without edges model of Chan and Vese to account for the ACF's particular structure. We employ level sets to represent the segmentation boundaries, and discretize in space by finite elements and in (artificial) time by finite differences. The approach is able to identify the ACF, their boundaries, and some of the internal crypts' orifices.
\end{abstract}

KEYWORDS: Image segmentation, active contours, level sets, endoscopic images.

\section{Introduction}

In the human colon's epithelium there are millions of invaginations (approximately 10 millions according to [25]) called crypts. Each crypt is a cylindrical tube, with a round opening in the top, directed at the lumen's colon, that contains different populations of cells $(\approx 300$ cells in total, with $\approx 15$ in perimeter and $\approx 20$ from the closed bottom to the orifice, see [15, 37]). These cells are aligned along the crypt wall: stems cells are believed to reside in the bottom of the crypt, transit cells along the middle part of the crypt axis and differentiated cells at the top of the crypt. In normal colonic crypts the cells renew completely each 3-4 days, through a programmed mechanism which includes the proliferation of cells, their migration along the crypt wall towards the top and their apoptosis, as they reach the top and the cell cycle is finished. If this programmed mechanism changes, disease may appear: the shape of the crypts change, they become aberrant crypts, aggregate in clusters and thus aberrant crypt foci (ACF for short) appear. Nowadays it is believed that ACF may represent preneoplastic lesions in the human colon, i.e., ACF may be the precursors of colorectal cancer (cf. $[4,6]$ ). This is one of the most frequent and malignant tumor in the world, and in Portugal it

Received June 7, 2008.

This work was partially supported by the UT Austin | Portugal Program. 
is the leading cause of cancer related death (cf. [18] for statistics in Europe and [21] in the United States). Therefore the study, analysis and evolution in vivo of $\mathrm{ACF}$ is a prominent issue in the medical community.

The ACF were first described in [5], after some experiments with rats, which were subjected to the administration of a carcinogenic agent (azoxymethane). A few years later, these lesions detected in rats were considered potential preneoplastic lesions in the murine colon, see [24]. Nowadays, human colonic ACF foci are defined as sets with one or more crypts, which stain darker with methylene blue and are larger than normal crypts, have a larger peri-cryptal zone and a thick epithelial lining (see also [31] for a classification of human ACF). Currently, ACF can de detected in vivo with magnification chromoscopic endoscopy (cf. [1, 20,34]). This is a medical endoscopic technique (that has a high patient acceptability) which combines chromoscopy and magnification. Chromoscopy consists in the application, by endoscopy, of colored substances in the colonic mucosa and subsequent visual analysis of the tissue stains in lesions (their location and features, as for example, the dimension, number, pattern, shape, etc.). By magnification it is meant an endoscopic diagnostic system, which enables the study and analysis of the crypt's orifices as well as micro-circulation. This term magnification includes two different concepts: high-resolution and amplification. The first is related to the ability to discriminate two objects or two points which are very close, whereas amplification is related to the possibility of increasing the whole size of the image. For an endoscope, the high-resolution depends on the density of the pixels incorporated in the chips, and the amplification depends on the existence of a lens integrated in the endoscope, which is controlled by the medical doctor (in some endoscopes the amplification of the lens can achieve 105 times). By way of illustration, the human eye can distinguish objects with 125 to 165 micron ( 1 micron is equal to $10^{-6}$ meter), while a high-resolution endoscope can discriminate objects with 10 to 17 micron. To detect details in the colonic mucosa it is necessary to distinguish parts of tissue with 10 micron (for instance, the dimension of a normal human crypt is approximately 74 and 433 micron, respectively, for the horizontal and vertical cross sections, see [19, 22]).

The current methods doctors use for assessing ACF patterns are somewhat subjective and not standardized. They are not computerized and rely only on direct medical observation. Through the endoscopic exam the medical doctor has a top view of the colon wall, and in particular the crypts' orifices, 
including their shapes, number, location. We note that, for each patient, the total number of $\mathrm{ACF}$ and the dimension of each $\mathrm{ACF}$, expressed (if this is possible) in terms of the number of crypt's orifices and these orifices' shapes, are most relevant for medical analysis. It would be very useful and helpful for medical doctors to have a reliable computerized and fast method for assessing the ACF's patterns images. This method would be able to identify the external boundary of each ACF, as well as, the crypts' orifices in its interior. This can be achieved with some image processing method. In an abstract framework this is a method (see [10]), which operates on a given acquired image (which could be degraded due to some external causes) and produces targeted features and patterns of this image. Mathematically, this corresponds to an inverse problem: in fact, the given acquired image is generated from the targeted features and the aim is to recover or detect precisely these features.

In this paper we focus on a particular image processing method, for assessing the ACF captured in vivo by endoscopy: image segmentation. This method consists in the partition of the given image into disjoint regions, representing distinct objects. Moreover, we use image segmentation methods based on partial differential equations, more exactly, active contours without edges and level set methods. These combine techniques of curve evolution (where the basic idea is to start with an initial curve in the image and to deform it to the boundaries of the objects in the image, and stop it there, see $[7,11,23]$ ), Mumford-Shah functional for image segmentation (an optimization problem to obtain a partition of the given image into distinct regions, see [26]) and level set methods (essentially these consist in considering the problem in a higher dimension, such that the evolving curve is the zero level set of an unknown function; these methods allow cusps, corners, and automatic topology changes, as merging and breaking curves, see [27, 28, 33]). We note that the expression "without edges" in "active contours without edges" refers to the fact that in these models it is not used any edge-detector function, based on the gradient of the given image, to identify the different objects (the "edges", in an image, are the boundaries of the distinct objects, corresponding to the places where these objects meet). This latter property allows the model to segment images where there are no clear gradient boundaries, which is often the case for ACF endoscopic images.

To be more precise, in this paper, we describe and apply to ACF endoscopic images, different active contour without edges models, existing in the 
literature (see $[11,9,8,38]$ ), for which we have introduced several modifications. One main modification concerns the numerical scheme we adopt for solving each one of these models. It is based on their weak variational formulations and finite elements discretizations (using the software COMSOL Multiphysics@ [12]); this differs from [11, 9, 8, 38], where a strong formulation is used (requiring higher regularity for the unknown level set function) and finite differences are used for the numerical approximations. In addition, we propose two new active contour without edges models. The first, is defined in section 2.2 and includes new terms, which represent specific ACF's features, which should be captured in the images and that were pointed out by medical doctors. The second, defined in section 2.3 , is a mixed regularized active contour without edges model, which intends to overcome the nonuniqueness issue (detrimental to optimization methods) that there are infinite level sets functions with the same zero level set. Furthermore, we also point out combined frameworks of the two new models and the previous ones. On the whole, all these models correspond to optimization problems, whose solution involves both PDE techniques (PDE is the abbreviation of partial differential equation), as for example, finite element discretizations, and numerical optimization methods. We believe that all the methods, used in the present paper, would perform even better and might give a much more accurate segmentation of the $\mathrm{ACF}$, if they incorporated specific features related to the ACF's endoscopy technique. In particular, the chromoscopy, alone, or maybe combined with material properties of the colon and the crypts. Our ongoing research pursues along these lines (see for instance the recent paper [3] for similar arguments in other context). The new model we propose in section 2.2 is a first attempt in this direction.

To the best of our knowledge there are no articles in the literature reporting image segmentation for in vivo ACF endoscopic images. However, we have found out four articles, which are, in some sense, close to this paper. The article [30] deals with image segmentation (using watershed morphological processing) of microscopic images of ACF, after resection. In [20] the ACF quantification and histological phenotype is done using a technique denominated by pixilation density mapping. In [14] it is used topographic segmentation for endoscopic capsule exams, but not for ACF images. The article [29] concerns automated image analysis tools for colorectal cancer.

We finish this introduction with the outline of the paper. In section 2 we describe several active contour without edges models, including two new ones 
proposed in sections 2.2 and 2.3, and the new numerical algorithm adopted for the solution of all these models. We report, in section 3, the results obtained for seven different human ACF endoscopic images, provided by the Faculty of Medicine of the University of Coimbra and the Department of Gastroenterology of University Hospital of Coimbra. All the segmentation results were done with the software Comsol Multiphysics@ [12] (in its implementation we incorporated the new numerical scheme detailed at the end of section 2.1). Finally some conclusions and future work are discussed in the last section.

\section{Active contours without edges models}

Let $\Omega$ be a bounded open set of $\mathbb{R}^{2}, u_{0}: \bar{\Omega} \longrightarrow \mathbb{R}$ be a given image and $\omega \subset \Omega$ a subset of $\Omega$, whose boundary is represented by the curve $C=\partial \omega$. Using an unified mathematical approach the active contours without edges models described in $[11,9,8,38]$ (we remark that $[9,8,38]$ can be considered extensions and variations of [11]) are optimization problems of the form

$$
\min _{(\phi, c)} F(\phi, c) \text {. }
$$

Here $c$ is an unknown constant vector with several components representing the distinct regions in the image $u_{0}$ we want to segment (say the subset $\omega$ ), and $\phi: \Omega \longrightarrow \mathbb{R}$ is an unknown function (the so-called level set function), representing the boundaries among these regions. That is, the set 0-level set, $\{\phi=0\}$, is the boundary $\partial \omega$ of $\omega$. The models $[11,9,8,38]$ differ in the definition of $F$, which always involves fitting terms (trying to fit $c$ to the given image $u_{0}$ ) and regularizing terms. But solving (1) amounts to resolve the necessary optimality conditions for the minimization of $F(\phi, c)$. This requires that the Gâteaux derivative $F^{\prime}(\phi, c)(\psi, s)$ vanishes for all admissible test functions $(\psi, s)$. Taking into account the specific definition of $F$, for the different models in $[11,9,8,38]$, these optimality conditions demonstrate that a minimum $(\phi, c)$ satisfies: $c$ is an explicit function of $\phi$, and $\phi$ is the solution of $\partial_{\phi} F(\phi, c)(\psi)=0$, for all admissible $\psi$. Due to this, the numerical scheme, used in $[11,9,8,38]$, is a two-step iterative scheme. Starting with an initial level set function $\phi^{0}$, the vector $c$ is computed and then its value is used to obtain the next level set function iterate $\phi$. This scheme is based totally on finite differences discretizations and and also on the strong formulation corresponding to the variational problem $\partial_{\phi} F(\phi, c)(\psi)=0$, which imposes more regularity on the unknown $\phi$. 
In this paper we propose a different numerical scheme to solve all these active contours without edges model, that differs from those implemented in $[11,9,8,38]$. It relies on finite element discretization for the space variable, and uses the weak and not the strong formulation of the optimality conditions. We point out these differences at the end of section 2.1. Moreover, in sections 2.2 and 2.3 we present two new active contours without edges models: conceptually they differ from [11] because they involve additional terms. In section 2.2 the new terms intend to capture specific features of the $\mathrm{ACF}$, while in the model of section 2.3, the two new terms are directly related to sophisticated optimization techniques. In addition we suggest combined frameworks of these new models with those defined in $[11,9,8,38]$. We remark that we have tested all the models with the new numerical scheme reported on this paper, on the ACF endoscopic images (see section 3).

To sum up the structure of this section is the following: for each one of the six active contours without edges models, and combined frameworks, we briefly define the minimization functional $F$, the meaning of the unknown $(\phi, c)$, point out the formulas for $c$ and $\partial_{\phi} F(\phi, c)(\psi)$, and explain the new numerical scheme. This latter is detailed in section 2.1 for one active contours without edges model, and for the subsequent five models we just comment on its adaptation.

2.1. The model of [11] and finite element approximation. For the active without edges model (ACWE model for short) introduced in [11]

$$
\begin{aligned}
F(\phi, c):= & \lambda^{+} \int_{\Omega}\left|u_{0}(x, y)-c^{+}\right|^{2} H(\phi(x, y)) d x d y \\
& +\lambda^{-} \int_{\Omega}\left|u_{0}(x, y)-c^{-}\right|^{2}(1-H(\phi(x, y))) d x d y \\
& +\mu \int_{\Omega} \delta_{0}(\phi(x, y))|\nabla \phi(x, y)| d x d y+\eta \int_{\Omega} H(\phi(x, y)) d x d y,
\end{aligned}
$$

where $\lambda^{+}>0, \lambda^{-}>0, \mu \geq 0$ and $\eta \geq 0$ are given fixed parameters, $c=\left(c^{+}, c^{-}\right), H(z):=1$ if $z \geq 0, H(z):=0$ if $z<0$ is the Heaviside function, and $\delta_{0}(z):=\frac{d}{d z} H(z)$ is the Dirac delta function in the sense of distributions.

The functional (2) is a modified version of the Mumford-Shah segmentation functional. It can be interpreted as to be looking for the best approximation in the $L^{2}$-sense to the given image $u_{0}$ among all functions that take only two values (denoted by $c^{+}$and $c^{-}$). In the right-hand side of (2) the first two integrals are fitting terms, the remaining two (related to the length of $C$ and area inside $C$ ) are regularizing terms. Then, the minimization problem (1), with $F$ defined by (2), corresponds to a partition of the image $u_{0}$ into two 
regions $c^{+}$and $c^{-}$, representing the averages of $u_{0}$ inside and outside $C$. The object $\omega$ to be detected or segmented is identified with one of the regions, the other object is the background, and $C$ will be the boundary of the object. It can be shown (cf. [27] page 124) that the parameter $\mu$ in the length term should be small if many small objects have to be detected and large if only large objects are to be detected.

Actually, in practise, for the minimization of $F(\phi, c)$, it is necessary to use in (2) a regularized version $H_{\varepsilon}$ of $H$ (i.e. a smooth approximation of $H$ ) and consequently an approximation $\delta_{\varepsilon}$ of $\delta_{0}$ (see also [16]). In [11] the choice is $\left.H_{\varepsilon}(z):=\frac{1}{\pi}\left(\arctan \left(\frac{z}{\varepsilon}\right)+\frac{1}{2 \varepsilon}\right)\right)$, with $\varepsilon=1$ regardless the resolution of the image, and $\delta_{\varepsilon}(z)=H_{\varepsilon}^{\prime}(z)=\frac{\varepsilon}{\pi\left(\varepsilon^{2}+z^{2}\right)}$. The unknown constants $c^{+}$and $c^{-}$are functions of $\phi$ given by

$$
c^{+}(\phi)=\frac{\int_{\Omega} u_{0} H_{\varepsilon}(\phi) d x d y}{\int_{\Omega} H_{\varepsilon}(\phi) d x d y}, \quad c^{-}(\phi)=\frac{\int_{\Omega} u_{0}\left(1-H_{\varepsilon}(\phi)\right) d x d y}{\int_{\Omega}\left(1-H_{\varepsilon}(\phi)\right) d x d y},
$$

which are, approximately, the averages of $u_{0}$ inside $(C):=\omega=\{\phi>0\}$ and outside $(C):=\Omega \backslash \omega=\{\phi<0\}$, respectively. On the other hand, $\phi$ is the solution of following the nonlinear variational equation, for any $\psi \in H^{1}(\Omega)$ (we recall that $H^{s}(\Omega)$, with $s \geq 1$, is the set of all functions in $L^{2}(\Omega)$, whose distributional derivative, up to the power $s$, belong to $L^{2}(\Omega)$ )

$$
\begin{aligned}
\partial_{\phi} F(\phi, c)(\psi)= & \int_{\Omega}\left(\eta+\lambda^{+}\left|u_{0}-c^{+}\right|^{2}-\lambda^{-}\left|u_{0}-c^{-}\right|^{2}\right) \delta_{\varepsilon}(\phi) \psi d x d y \\
& +\int_{\Omega} \mu\left(\delta_{\varepsilon}^{\prime}(\phi)|\nabla \phi| \psi+\delta_{\varepsilon}(\phi) \frac{\nabla \phi \cdot \nabla \psi}{|\nabla \phi|}\right) d x d y=0 .
\end{aligned}
$$

If $\phi \in H^{2}(\Omega)$, a Green formula can be applied to the term $\int_{\Omega} \mu \delta_{\varepsilon}(\phi) \frac{\nabla \phi \cdot \nabla \psi}{|\nabla \phi|} d x d y$, and then, (4) becomes

$$
\begin{aligned}
\partial_{\phi} F(\phi, c)(\psi)= & \int_{\Omega}\left(\eta+\lambda^{+}\left|u_{0}(x, y)-c^{+}\right|^{2}-\lambda^{-}\left|u_{0}(x, y)-c^{-}\right|^{2}\right) \delta_{\varepsilon}(\phi) \psi d x d y \\
& \left.-\int_{\Omega} \mu \operatorname{div}\left(\frac{\nabla \phi}{|\nabla \phi|}\right) \delta_{\varepsilon}(\phi) \psi d x d y+\int_{\partial \Omega} \mu \frac{\delta_{\varepsilon}(\phi)}{|\nabla \phi|}\right) \frac{\partial \phi}{\partial n} \psi d x d y=0,
\end{aligned}
$$

where $\frac{\partial \phi}{\partial n}$ is the normal derivative of $\phi$ in the boundary $\partial \Omega$ of $\Omega$.

An usual methodology, to solve $\partial_{\phi} F(\phi, c)(\psi)=0$, for any admissible $\psi$, is to introduce an artificial time parameter $t \geq 0$ and search for the stationary solution $\phi(t, x, y)$ with respect to the variable $t$. This means, to solve, for each $t$, the problem

$$
\int_{\Omega} \frac{\partial \phi}{\partial t} \psi d x d y=0 \quad \text { with } \quad \int_{\Omega} \frac{\partial \phi}{\partial t} \psi d x d y=\partial_{\phi} F(\phi, c)(\psi),
$$


for all $\psi \in H^{1}(\Omega)$. Then, a numerical scheme which can be used to approximate the solution of the ACWE model is a two-step scheme, where in the first step the values of the unknowns average regions $c^{+}$and $c^{-}$are computed by formulas (3) and in the second step the minimization of $F(., c)$ is performed updating the level set function $\phi$. The different steps of this method are:

\section{NumericAl SCHEME}

- Step 1 - Initialize with $\phi^{0}$, and $n=0$.

- Step 2- For $n \geq 0$, compute $c^{n}=\left(c^{+}\left(\phi^{n}\right), c^{-}\left(\phi^{n}\right)\right)$ by (3).

- Step 3-Given $\phi^{n}$, solve $\partial_{\phi} F\left(\phi, c^{n}\right)(\psi)=0$ by (4) (or by (6), if the artificial variable $t$ is introduced), to obtain $\phi^{n+1}$.

- Step 4-Stopping criterium :

- If formula (4) is used, stop when $\left\|\phi^{n+1}-\phi^{n}\right\|_{H^{1}(\Omega)} \leq$ tol with tol a given tolerance, and $c^{+}\left(\phi^{n+1}\right) \approx c^{+}\left(\phi^{n}\right)$ and $c^{-}\left(\phi^{n+1}\right) \approx c^{-}\left(\phi^{n}\right)$.

- If formula (6) is used, stop when $\frac{\partial \phi^{n+1}}{\partial t} \approx 0$, that is, the solution $\phi^{n+1}$ is stationary (in this case we set in Step 1, for $t=0$, $\left.\phi(0, x, y)=\phi^{0}(x, y)\right)$, and $c^{+}\left(\phi^{n+1}\right) \approx c^{+}\left(\phi^{n}\right)$ and $c^{-}\left(\phi^{n+1}\right) \approx$ $c^{-}\left(\phi^{n}\right)$.

In [11], Step 3 is solved by using a finite differences implicit scheme, which approximates the strong formulation of $(6)$, with $\partial_{\phi} F(\phi, c)(\psi)$ defined by $(5)$ requiring $\phi \in H^{2}(\Omega)$. In this paper we adopt a different numerical method. We always use finite elements for the discretization in space and the numerical algorithm chosen to solve Step 3 can be either a direct optimization algorithm (when the extra variable $t$ is not introduced) or an implicit scheme which combines the finite element discretization in space with an approximation of the time derivative. Furthermore for the finite element discretization we always use the variational formulation (4) and not (5), which needs more regularity on the unknown $\phi$. We give now the description of the implicit scheme, we used for segmenting the ACF endoscopic images. Considering a finite element mesh and denoting by the same letter $\phi$ the finite element approximation of $\phi$, then the right formula in (6) becomes

$$
M \frac{\partial \phi}{\partial t}=L(\phi, c)
$$

Here $M$ and $L$ are, respectively, the mass and stiffness matrices ( $L$ is the finite element discretization of $\partial_{\phi} F(\phi, c)$ defined in (4), which is a nonlinear function of $\phi$ ). Afterwards, we choose a Taylor expansion of first order for the right hand side of (7) (starting with the initial guess $\phi^{n}$ and freezing the 
dependence of $c=\left(c^{+}, c^{-}\right)$on $\left.\phi^{n}\right)$, and finite differences for the left hand side. Hence, the approximation of (7) becomes

$$
M \frac{\phi^{n+1}-\phi^{n}}{\triangle t}=K\left(\phi^{n}, c^{+}\left(\phi^{n}\right), c^{-}\left(\phi^{n}\right)\right)\left(\phi^{n+1}-\phi^{n}\right)+L\left(\phi^{n}, c^{+}\left(\phi^{n}\right), c^{-}\left(\phi^{n}\right)\right)
$$

where $\Delta t$ is the time step and $K$ is the Jacobian (stiffness matrix) of $L$, with respect to the first argument $\phi$. Then, (8) is equivalent to the following linear system

$$
\begin{aligned}
& \left(\frac{M}{\triangle t}-K\left(\phi^{n}, c^{+}\left(\phi^{n}\right), c^{-}\left(\phi^{n}\right)\right)\right) \phi^{n+1}= \\
& \quad\left(\frac{M}{\triangle t}-K\left(\phi^{n}, c^{+}\left(\phi^{n}\right), c^{-}\left(\phi^{n}\right)\right)\right) \phi^{n}+L\left(\phi^{n}, c^{+}\left(\phi^{n}\right), c^{-}\left(\phi^{n}\right)\right)
\end{aligned}
$$

whose unknown is $\phi^{n+1}$. Actually, in the numerical realization (see section 3), we do not update $c^{+}\left(\phi^{n}\right)$ and $c^{-}\left(\phi^{n}\right)$ in each time step but only after a fixed number of time steps. Moreover, the time step $\Delta t$ is not fixed but subject to variation. Since we are only interested in the convergence to a stationary state we increase the size of the time steps during the iteration. In addition, and because the norm of the gradient $|\nabla \phi|$ appears in a denominator in $L\left(\phi, c^{+}\left(\phi^{n}\right), c^{-}\left(\phi^{n}\right)\right)$ (see (4)), we replace $\frac{1}{|\nabla \phi|}$ by $\frac{1}{\left|\nabla \phi^{n}\right|}$ (this change in the definition of $L$ is also used in the computation of the Jacobian matrix $K$ of $L)$ and keep it fixed exactly during the same number of time steps as for $c^{+}\left(\phi^{n}\right)$ and $c^{-}\left(\phi^{n}\right)$.

Summarizing, we have two loops. An outer loop where we update $c^{+}\left(\phi^{n}\right)$, $c^{-}\left(\phi^{n}\right)$ and $\left|\nabla \phi^{n}\right|$. An inner loop, which is defined by (9), where $c^{+}\left(\phi^{n}\right)$, $c^{-}\left(\phi^{n}\right)$ and $\frac{1}{\left|\nabla \phi^{n}\right|}$ are kept fixed, and that updates $\phi^{n}$ to $\phi_{k}^{n}$ in each inner iteration $k$ (the total number $m$ of time steps, with increasing magnitudes, is fixed a priori). So, for each outer iterate $n$, the new iterate $\phi^{n+1}$ in Step 2 entering in the outer loop, is the final of the inner loop, that is, $\phi^{n+1}=\phi_{m}^{n}$. In the inner loop, for the Newton-type approximation, the initial guess is always $\phi^{0}$ in the first iteration, while in the subsequent iterations it is the previous iterate (i.e. $\phi_{k-1}^{n}$ in iteration $k$ of the inner loop).

2.2. A modified model. Using the a priori medical knowledge that the crypts' boundaries stain darker than normal crypts and that in general inside each foci, the shape of the crypts's orifices follows a similar pattern, we have decided to modify the original ACWE model, described in the previous section, by including two terms that express these features. More precisely, 
we choose the following formulation (compare to (1) with $F$ defined by (2))

$$
\min _{(\phi, c, \iota, \kappa)} F(\phi, c, \iota, \kappa)
$$

with

$$
\begin{aligned}
F(\phi, c, \iota, \kappa):= & \lambda^{+} \int_{\{\phi>0\}}\left|u_{0}-c^{+}\right|^{2} d x d y+\lambda^{-} \int_{\{\phi<0\}}\left|u_{0}-c^{-}\right|^{2} d x d y+ \\
& \mu \int_{\{\phi=0\}} 1 d x d y+\eta \int_{\{\phi>0\}} 1 d x d y+ \\
& \rho_{\iota} \int_{\{\phi=0\}}\left|u_{0}-\iota\right|^{2} d x d y+\rho_{\kappa} \int_{\{\phi=0\}}\left|\operatorname{div}\left(\frac{\nabla u_{0}}{\left|\nabla u_{0}\right|}\right)-\kappa\right|^{2} d x d y .
\end{aligned}
$$

The new unknown constants, $\iota$ and $\kappa$, represent constant approximations of the image $u_{0}$ and of its curvature, respectively, on the 0 -level set of $\phi$. The new fitting terms are weighted with two small positive parameters $\rho_{\iota}$ and $\rho_{\kappa}$, in order not not change too much the original problem. Of course we have

$$
\begin{aligned}
& \int_{\{\phi=0\}}\left|u_{0}-\iota\right|^{2} d x d y=\int_{\Omega}\left|u_{0}-\iota\right|^{2} \delta_{0}(\phi)|\nabla \phi| d x d y \\
& \int_{\{\phi=0\}}\left|\operatorname{div}\left(\frac{\nabla u_{0}}{\left|\nabla u_{0}\right|}\right)-\kappa\right|^{2} d x d y=\int_{\Omega}\left|\operatorname{div}\left(\frac{\nabla u_{0}}{\left|\nabla u_{0}\right|}\right)-\kappa\right|^{2} \delta_{0}(\phi)|\nabla \phi| d x d y .
\end{aligned}
$$

Computing the Gâteaux derivative of $F$ we obtain that $c^{+}$and $c^{-}$are given by (3) and $\iota$ and $\kappa$ are functions of $\phi$ defined by

$$
\begin{aligned}
& \iota(\phi)=\frac{\int_{\Omega} u_{0} \delta_{\varepsilon}(\phi)|\nabla \phi| d x d y}{\int_{\Omega} \delta_{\varepsilon}(\phi)|\nabla \phi| d x d y} \approx \frac{\int_{\{\phi=0\}} u_{0} d s}{\int_{\{\phi=0\}} 1 d s}, \\
& \kappa(\phi)=\frac{\int_{\Omega} \operatorname{div}\left(\frac{\nabla u_{0}}{\left|\nabla u_{0}\right|}\right) \delta_{\varepsilon}(\phi)|\nabla \phi| d x d y}{\int_{\Omega} \delta_{\varepsilon}(\phi)|\nabla \phi| d x d y} \approx \frac{\int_{\{\phi=0\}} \operatorname{div}\left(\frac{\nabla u_{0}}{\left|\nabla u_{0}\right|}\right) d s}{\int_{\{\phi=0\}} 1 d s} .
\end{aligned}
$$

Thus, $\iota$ represents the approximation of the average of the image $u_{0}$ on the 0 -level set $\{\phi=0\}$ and $\kappa$ the approximation of the average curvature of the level sets of $u_{0}$ on $\{\phi=0\}$. Moreover, keeping $(c, \iota, \kappa)$ fixed, the Gâteaux derivative $\partial_{\phi} F(\phi, c, \iota, \kappa)(\psi)$ is equal to (compare with (4))

$$
\begin{gathered}
\partial_{\phi} F(\phi, c, \iota, \kappa)(\psi)=\int_{\Omega}\left(\eta+\lambda^{+}\left|u_{0}-c^{+}\right|^{2}-\lambda^{-}\left|u_{0}-c^{-}\right|^{2}\right) \delta_{\varepsilon}(\phi) \psi d x d y+ \\
\int_{\Omega}\left(\mu+\rho_{\iota}\left|u_{0}-\iota\right|^{2}+\rho_{\kappa}\left|\operatorname{div}\left(\frac{\nabla u_{0}}{\left|\nabla u_{0}\right|}\right)-\kappa\right|^{2}\right)\left(\delta_{\varepsilon}^{\prime}(\phi)|\nabla \phi| \psi+\delta_{\varepsilon}(\phi) \frac{\nabla \phi \cdot \nabla \psi}{|\nabla \phi|}\right) d x d y \\
=0,
\end{gathered}
$$

for any $\psi \in H^{1}(\Omega)$. Clearly an analogous numerical scheme, like the one indicated on page 8 applies. But now in step 2, and in the outer loop, in 
addition to the computation of $c^{+}\left(\phi^{n}\right)$ and $c^{-}\left(\phi^{n}\right)$ by (3), we also have to evaluate $\iota\left(\phi^{n}\right)$ and $\kappa\left(\phi^{n}\right)$ by $(13)$.

From the numerical point of view, the difference between this new model and the one of the previous section, resides in the definition of the coefficient of the length term. In the model of section 2.1, this coefficient is constant and equal to $\mu$, while here its value varies, being equal to

$$
\mu+\rho_{\iota}\left|u_{0}-\iota\left(\phi^{n}\right)\right|^{2}+\rho_{\kappa}\left|\operatorname{div}\left(\frac{\nabla u_{0}}{\left|\nabla u_{0}\right|}\right)-\kappa\left(\phi^{n}\right)\right|^{2}
$$

in each iteration $n$ of the outer loop.

2.3. A mixed regularized model. We remark for the model of section 2.1 , there is an infinite number of level set functions $\phi$ with the same zero level set. To overcome this nonuniqueness issue we decided to regularize the model by adding a term, depending on $\nabla \phi$ which implicitly imposes a unique value to the gradient of the level set function (cf. [13] where a similar regularization technique was used for shape optimization problems; there the objective function is the nonlinear least square Heaviside distance between the target shape and the developing shape). Now, the new minimization functional is

$$
\begin{aligned}
F(\phi, c):= & \lambda^{+} \int_{\{\phi>0\}}\left|u_{0}-c^{+}\right|^{2} d x d y+\lambda^{-} \int_{\{\phi<0\}}\left|u_{0}-c^{-}\right|^{2} d x d y \\
& +\mu \int_{\{\phi=0\}} 1 d s+\eta \int_{\{\phi>0\}} 1 d s \\
& +\frac{\beta}{4} \int_{\Omega}\left(|\nabla \phi|^{2}-1\right)^{2} d x d y+\frac{\alpha}{2} \int_{\Omega}|\nabla \phi|^{2} d x d y,
\end{aligned}
$$

where $\beta \geq 0$ and $\alpha \geq 0$ are two positive fixed parameters. We observe that a Tikhonov regularization is obtained with $\beta=0$. When $\beta \gg \alpha$, then the model tends to oblige the level set function $\phi$ to be closed to a signed distance function, that is, $|\nabla \phi|=1$, everywhere. Now, the Gâteaux derivative $\partial_{\phi} F\left(\phi, c^{+}, c^{-}\right)(\psi)$ of $F$ with respect to $\phi$ becomes (compare to (4))

$$
\begin{aligned}
\partial_{\phi} F(\phi, c)(\psi)= & \int_{\Omega}\left(\eta+\lambda^{+}\left|u_{0}-c^{+}\right|^{2}-\lambda^{-}\left|u_{0}-c^{-}\right|^{2}\right) \delta_{\varepsilon}(\phi) \psi d x d y \\
& +\int_{\Omega} \mu\left(\delta_{\varepsilon}^{\prime}(\phi)|\nabla \phi| \psi+\delta_{\varepsilon}(\phi) \frac{\nabla \phi \cdot \nabla \psi}{|\nabla \phi|}\right) d x d y \\
& +\int_{\Omega}(\beta(\nabla \phi \cdot \nabla \phi-1)+\alpha) \nabla \phi \cdot \nabla \psi d x d y=0 .
\end{aligned}
$$

Clearly, the numerical scheme of page 8 applies also to this new minimization problem, with $\partial_{\phi} F\left(\phi, c^{+}, c^{-}\right)(\psi)$ defined by (16). 
2.4. A vector-valued model. Suppose now that the given image $u_{0}$ is a vector-valued image, with $n$-channels, for instance a colored image, as a RGB image with the red, green and blue channels, or a vector-valued image obtained from a textured image. We note that ACF endoscopic images exhibit these features. Then, $u_{0}=\left(u_{0 i}\right)_{i=1}^{n}=\left(u_{01}, u_{02}, \ldots, u_{0 n}\right)$, where $u_{0 i}: \bar{\Omega} \longrightarrow \mathbb{R}$ is the $i t h$-component (or equivalently the $i t h$-channel) of image $u_{0}$. Theoretically and ideally, each channel would contain different information of the same image (for instance, in the case where there are objects with different missing parts in different channels). Then the extension of the original ACWE's functional defined in (2) consists in replacing the fitting error of the scalar image, by the sum of the fitting errors over each component of the vector-valued image. Let $c:=\left(c^{+}, c^{-}\right)$and $c^{+}:=\left(c_{i}^{+}\right)_{i=1}^{n}=\left(c_{1}^{+}, c_{2}^{+}, \ldots, c_{n}^{+}\right)$ and $c^{-}:=\left(c_{i}^{-}\right)_{i=1}^{n}=\left(c_{1}^{-}, c_{2}^{-}, \ldots, c_{n}^{-}\right)$, where $c_{i}^{+}$and $c_{i}^{-}$represent the two unknown constant approximations of channel $u_{0 i}$, inside and outside the evolving contour. Hence, the generalization of (2) is (see [9])

$$
\begin{aligned}
F(\phi, c):= & \int_{\Omega} \frac{1}{n} \sum_{i=1}^{n} \lambda_{i}^{+}\left|u_{0 i}(x, y)-c_{i}^{+}\right|^{2} H(\phi(x, y)) d x d y \\
& +\int_{\Omega} \frac{1}{n} \sum_{i=1}^{n} \lambda_{i}^{+}\left|u_{0 i}(x, y)-c_{i}^{-}\right|^{2}(1-H(\phi(x, y))) d x d y \\
& +\mu \int_{\Omega} \delta_{0}(\phi(x, y))|\nabla \phi(x, y)| d x d y+\eta \int_{\Omega} H(\phi(x, y)) d x d y,
\end{aligned}
$$

where $\lambda^{+}=\left(\lambda^{+}\right)_{i=1}^{n}=\left(\lambda_{1}^{+}, \lambda_{2}^{+}, \ldots, \lambda_{n}^{+}\right)$and $\lambda^{-}=\left(\lambda^{-}\right)_{i=1}^{n}=\left(\lambda_{1}^{-}, \lambda_{2}^{-}, \ldots, \lambda_{n}^{-}\right)$, with $\lambda_{i}^{+}$and $\lambda_{i}^{-}$fixed positive scalars weighting the fitting terms.

Fixing $\phi$ and minimizing $F(\phi, c)$ with respect to the unknown constants $c_{i}^{+}$ and $c_{i}^{-}$, for $i=1, \ldots, n$, we obtain

$$
c_{i}^{+}(\phi)=\frac{\int_{\Omega} u_{0 i} H_{\varepsilon}(\phi) d x d y}{\int_{\Omega} H_{\varepsilon}(\phi) d x d y}, \quad c_{i}^{-}(\phi)=\frac{\int_{\Omega} u_{0 i}\left(1-H_{\varepsilon}(\phi)\right) d x d y}{\int_{\Omega}\left(1-H_{\varepsilon}(\phi)\right) d x d y},
$$

which are approximately the averages of $u_{0 i}$ on $\{\phi>0\}$ and on $\{\phi<0\}$, respectively. On the other hand, keeping $c^{+}$and $c^{-}$constants and minimizing $F(\phi, c)$ with respect to $\phi$, the Gâteaux derivative $\partial_{\phi} F(\phi, c)(\psi)$ of $F$ with respect to $\phi$ verifies (compare with (4))

$$
\begin{aligned}
\partial_{\phi} F(\phi, c)(\psi)= & \int_{\Omega}\left(\eta+\frac{1}{n} \sum_{i=1}^{n}\left(\lambda_{i}^{+}\left|u_{0 i}(x, y)-c_{i}^{+}\right|^{2}-\lambda_{i}^{-}\left|u_{0 i}(x, y)-c_{i}^{-}\right|^{2}\right)\right) \delta_{\varepsilon}(\phi) \psi d x d y \\
& +\int_{\Omega} \mu\left(\delta_{\varepsilon}^{\prime}(\phi)|\nabla \phi| \psi+\delta_{\varepsilon}(\phi) \frac{\nabla \phi \cdot \nabla \psi}{|\nabla \phi|}\right) d x d y=0,
\end{aligned}
$$

for any test function $\psi \in H^{1}(\Omega)$. Again the numerical scheme of page 8 applies with the following change in step 2: compute $c_{i}^{+}\left(\phi^{n}\right)$ and $c_{i}^{-}\left(\phi^{n}\right)$ by (18), for $i=1, \ldots, n$. 
Clearly we can modify this vector-valued model, as indicated in section 2.2 , by adding to the functional (17) the following new fitting terms for each channel (compare with (11))

$$
\frac{1}{n} \sum_{i=1}^{n} \rho_{\iota_{i}} \int_{\{\phi=0\}}\left|u_{0 i}-\iota_{i}\right|^{2} d x d y+\frac{1}{n} \sum_{i=1}^{n} \rho_{\kappa_{i}} \int_{\{\phi=0\}}\left|\operatorname{div}\left(\frac{\nabla u_{0 i}}{\left|\nabla u_{0 i}\right|}\right)-\kappa_{i}\right|^{2} d x d y .
$$

Here $\iota=\left(\iota_{1}, \ldots, \iota_{n}\right)$ and $\kappa=\left(\kappa_{1}, \ldots, \kappa_{n}\right)$ are unknown vectors and $\rho_{\iota_{i}}, \rho_{\kappa_{i}}$ are given positive parameters. For each $i=1, \ldots, n, \iota_{i}$ and $\kappa_{i}$ represent, respectively, the average of the channel $u_{0 i}$ and the average curvature of the level sets of $u_{0 i}$, on the 0 -level set $\{\phi=0\}$.

We can also define a mixed regularized vector-valued ACWE model, as in section 2.3, by adding, to the functional (17), the terms (compare with (15))

$$
\frac{\beta}{4} \int_{\Omega}\left(|\nabla \phi|^{2}-1\right)^{2} d x d y+\frac{\alpha}{2} \int_{\Omega}|\nabla \phi|^{2} d x d y
$$

2.5. A convex model. The problem $\min _{\phi} F(\phi, c)$, of section 2.1 , is a nonconvex minimization problem, for $c=\left(c^{+}, c^{-}\right)$fixed. In [8], it is shown that this minimization problem can be equivalently reformulated as a convex unconstrained minimization problem. More precisely, let $\lambda^{+}=\lambda^{-}=\lambda$ in the definition (2) of $F$, then for fixed $\left(c^{+}, c^{-}\right)$, the problem $\min _{\phi} F(\phi, c)$ is equivalent to

$$
\begin{aligned}
\min _{\phi}\left(\int_{\Omega} \lambda\right. & \left(\left|u_{0}(x, y)-c^{+}\right|^{2}-\left|u_{0}(x, y)-c^{-}\right|^{2}\right) d x d y \\
& \left.+\int_{\Omega}(\mu|\nabla \phi(x, y)|+\eta \phi(x, y)) d x d y+\int_{\Omega} \theta p(\phi) d x d y\right)
\end{aligned}
$$

where $p(\xi):=\max \left\{0,2\left|\xi-\frac{1}{2}\right|-1\right\}$, provided that $\theta>\frac{\lambda}{2} \|\left|u_{0}(x, y)-c^{+}\right|^{2}-$ $\left|u_{0}(x, y)-c^{-}\right|^{2} \|_{L^{\infty}(\Omega)}$. Thus $p($.$) is an exact penalty term, which constrains$ the minimizer $\phi$ to vary in $[0,1]$. Denoting by $F_{p}(\phi, c)$ the objective functional in $(20)$, the necessary optimal condition requires that at the minimum $\phi$, the Gâteaux derivative $\partial_{\phi} F_{p}(\phi, c)(\psi)=0$, for any $\psi \in H^{1}(\Omega)$, where

$$
\begin{aligned}
\partial_{\phi} F_{p}(\phi, c)(\psi)= & \int_{\Omega}\left(\eta+\lambda^{+}\left|u_{0}-c^{+}\right|^{2}-\lambda^{-}\left|u_{0}-c^{-}\right|^{2}\right) \psi d x d y \\
& +\int_{\Omega} \mu \delta_{\varepsilon}(\phi) \frac{\nabla \phi \cdot \nabla \psi}{|\nabla \phi|} d x d y+\int_{\Omega} \theta p^{\prime}(\phi) \psi d x d y
\end{aligned}
$$


with $p^{\prime}($.$) the derivative of the penalty. Once again the numerical scheme of$ page 8 can be used where in step 3 we replace the objective functional by $F$ by $F_{p}$ and solve the equation $\partial_{\phi} F_{p}(\phi, c)\left(\phi^{n}\right)=0$. We note that in the numerical realization $p$ is replaced by a regularized penalty $p_{b}$, where $b$ is a positive real small constant. In section 3 we chose $p_{b}(z)=\frac{z^{2}}{b}$, for $z \leq 0, p_{b}(z)=0$, for $0<z<1$, and $p_{b}(z)=\frac{z^{2}}{2 b}-\frac{z}{b}-\frac{1}{2 b}$, for $z \geq 1$. This reformulation, as a non convex unconstrained minimization problem, also applies to the modified model or to the vector-valued model defined in sections 2.2 and 2.4. Moreover, the minimization problem (20) can be regularized as indicated in section 2.3.

2.6. A multi-phase model. The main idea, in the model [11], is to search for a partition of the given image $u_{0}$ in two-phases (or two regions) one representing the object to be detected and the other the background. In [38] it is proposed a variant of it where the aim is to look for a decomposition of the image in several regions, using, not only one, but several different level set functions. It is a multi-phase level set model for image segmentation, based again on the Mumford-Shah model, for piecewise constant or piecewise smooth optimal approximations. Here we briefly describe the case of piecewise constant segmentation with four regions, that is, a four-phase model. We note this multi-phase model is of particular interest for the ACF endoscopic images, since one of the aims is to extract the maximum of valuable and relevant information from the medical images. Now the minimization functional $F$ is

$$
\begin{aligned}
F(\phi, c):= & \lambda_{11} \int_{\Omega}\left|u_{0}-c_{11}\right|^{2} H_{\varepsilon}\left(\phi_{1}\right) H_{\varepsilon}\left(\phi_{2}\right) d x d y+ \\
& \lambda_{10} \int_{\Omega}\left|u_{0}-c_{10}\right|^{2} H_{\varepsilon}\left(\phi_{1}\right)\left(1-H_{\varepsilon}\left(\phi_{2}\right)\right) d x d y+ \\
& \lambda_{01} \int_{\Omega}\left|u_{0}-c_{01}\right|^{2}\left(1-H_{\varepsilon}\left(\phi_{1}\right)\right) H_{\varepsilon}\left(\phi_{2}\right) d x d y+ \\
& \lambda_{00} \int_{\Omega}\left|u_{0}-c_{00}\right|^{2}\left(1-H_{\varepsilon}\left(\phi_{1}\right)\right)\left(1-H_{\varepsilon}\left(\phi_{2}\right)\right) d x d y+ \\
& \mu_{1} \int_{\Omega} \delta_{\varepsilon}\left(\phi_{1}\right)\left|\nabla \phi_{1}\right| d x d y+\eta_{1} \int_{\Omega} H_{\varepsilon}\left(\phi_{1}\right) d x d y+ \\
& \mu_{2} \int_{\Omega} \delta_{\varepsilon}\left(\phi_{2}\right)\left|\nabla \phi_{2}\right| d x d y+\eta_{2} \int_{\Omega} H_{\varepsilon}\left(\phi_{2}\right) d x d y .
\end{aligned}
$$

Here, $\phi=\left(\phi_{1}, \phi_{2}\right)$ is the unknown level set function vector, with $\phi_{i}: \Omega \longrightarrow$ $\mathbb{R}$, for $i=1,2, \lambda_{i j}$, for $i, j=0,1$, and $\mu_{i}, \eta_{i}$, for $i, j=1,2$, are given positive parameters, and $c=\left(c_{11}, c_{10}, c_{01}, c_{00}\right)$ is an unknown vector whose 
components are the four regions of the image. By computing the Gâteaux derivative of $F(\phi, c)$ with $\phi$ kept fixed, we have

$$
\begin{aligned}
& c_{11}(\phi)=\frac{\int_{\Omega} u_{0} H_{\varepsilon}\left(\phi_{1}\right) H_{\varepsilon}\left(\phi_{2}\right) d x d y}{\int_{\Omega} H_{\varepsilon}\left(\phi_{1}\right) H_{\varepsilon}\left(\phi_{2}\right) d x d y}, \quad c_{10}(\phi)=\frac{\int_{\Omega} u_{0} H_{\varepsilon}\left(\phi_{1}\right)\left(1-H_{\varepsilon}\left(\phi_{2}\right)\right) d x d y}{\int_{\Omega} H_{\varepsilon}\left(\phi_{1}\right)\left(1-H_{\varepsilon}\left(\phi_{2}\right)\right) d x d y}, \\
& c_{01}(\phi)=\frac{\int_{\Omega} u_{0}\left(1-H_{\varepsilon}\left(\phi_{1}\right)\right) H_{\varepsilon}\left(\phi_{2}\right) d x d y}{\int_{\Omega}\left(1-H_{\varepsilon}\left(\phi_{1}\right)\right) H_{\varepsilon}\left(\phi_{2}\right) d x d y}, \quad c_{00}(\phi)=\frac{\int_{\Omega} u_{0}\left(1-H_{\varepsilon}\left(\phi_{1}\right)\right)\left(1-H_{\varepsilon}\left(\phi_{2}\right)\right) d x d y}{\int_{\Omega}\left(1-H_{\varepsilon}\left(\phi_{1}\right)\right)\left(1-H_{\varepsilon}\left(\phi_{2}\right)\right) d x d y},
\end{aligned}
$$

which are approximately the averages of $u_{0}$ on $\left\{\phi_{1}>0\right\} \bigcap\left\{\phi_{2}>0\right\}$, on $\left\{\phi_{1}>0\right\} \cap\left\{\phi_{2}<0\right\}$, on $\left\{\phi_{1}<0\right\} \bigcap\left\{\phi_{2}>0\right\}$ and on $\left\{\phi_{1}<0\right\} \cap\left\{\phi_{2}<0\right\}$, respectively.

Likewise, keeping the four-phase vector $c$ fixed, and minimizing $F(\phi, c)$ with respect to $\phi=\left(\phi_{1}, \phi_{2}\right)$, the Gâteaux derivative $\partial_{\phi} F(\phi, c)(\psi)$, for any $\psi=\left(\psi_{1} \psi_{2}\right) \in\left[H^{1}(\Omega]^{2}\right.$, must verify (compare with (4))

$$
\begin{aligned}
\partial_{\phi} F(\phi, c)(\psi)= & \int_{\Omega}\left(\eta_{1}+\lambda_{11}\left|u_{0}-c_{11}\right|^{2}-\lambda_{10}\left|u_{0}-c_{10}\right|^{2}\right) H_{\varepsilon}\left(\phi_{2}\right) \delta_{\varepsilon}\left(\phi_{1}\right) \psi_{1} d x d y \\
& +\int_{\Omega}\left(\lambda_{01}\left|u_{0}-c_{01}\right|^{2}-\lambda_{00}\left|u_{0}-c_{00}\right|^{2}\right)\left(1-H_{\varepsilon}\left(\phi_{2}\right)\right) \delta_{\varepsilon}\left(\phi_{1}\right) \psi_{1} d x d y \\
& +\int_{\Omega} \mu_{1}\left(\delta_{\varepsilon}^{\prime}\left(\phi_{1}\right)\left|\nabla \phi_{1}\right| \psi_{1}+\delta_{\varepsilon}\left(\phi_{1}\right) \frac{\nabla \phi_{1} \cdot \nabla \psi_{1}}{\left|\nabla \phi_{1}\right|}\right) d x d y \\
& +\int_{\Omega}\left(\eta_{2}+\lambda_{11}\left|u_{0}-c_{11}\right|^{2}-\lambda_{10}\left|u_{0}-c_{10}\right|^{2}\right) H_{\varepsilon}\left(\phi_{1}\right) \delta_{\varepsilon}\left(\phi_{2}\right) \psi_{2} d x d y \\
& +\int_{\Omega}\left(\lambda_{01}\left|u_{0}-c_{01}\right|^{2}-\lambda_{00}\left|u_{0}-c_{00}\right|^{2}\right)\left(1-H_{\varepsilon}\left(\phi_{1}\right)\right) \delta_{\varepsilon}\left(\phi_{2}\right) \psi_{2} d x d y \\
& +\int_{\Omega} \mu_{2}\left(\delta_{\varepsilon}^{\prime}\left(\phi_{2}\right)\left|\nabla \phi_{2}\right| \psi_{2}+\delta_{\varepsilon}\left(\phi_{2}\right) \frac{\nabla \phi_{2} \cdot \nabla \psi_{2}}{\left|\nabla \phi_{2}\right|}\right) d x d y=0 .
\end{aligned}
$$

As mentioned before (see (6)), the artificial time parameter $t \geq 0$ can be introduced to solve (24) and thus formula (6) becomes, for any $\psi=\left(\psi_{1}, \psi_{2}\right) \in$ $\left[H^{1}(\Omega]^{2}\right.$

$$
\begin{aligned}
& \int_{\Omega}\left(\frac{\partial \phi_{1}}{\partial t} \psi_{1}+\frac{\partial \phi_{2}}{\partial t} \psi_{2}\right) d x d y=0 \quad \text { with, } \\
& \int_{\Omega} \frac{\partial \phi_{1}}{\partial t} \psi_{1} d x d y=\partial_{\phi} F(\phi, c)\left(\psi_{1}, 0\right), \quad \int_{\Omega} \frac{\partial \phi_{2}}{\partial t} \psi_{2} d x d y=\partial_{\phi} F(\phi, c)\left(0, \psi_{2}\right) .
\end{aligned}
$$

The numerical scheme described on page 8 can again be applied to this fourphase model with the following changes in steps 2 and 3 :

- Step 2-Compute $c_{i j}\left(\phi^{n}\right)$ by $(23)$.

- Step 3-Given $\phi^{n}=\left(\phi_{1}^{n}, \phi_{2}^{n}\right)$ solve $\partial_{\phi} F\left(\phi, c\left(\phi^{n}\right)\right)(\psi)$ by $(24)$, or alternatively, introduce an artificial time variable $t$ and solve, sequentially, 
the two variational problems to obtain, firstly $\phi_{1}^{n+1}$ and then $\phi_{2}^{n+1}$

$$
\begin{array}{ll}
\int_{\Omega} \frac{\partial \phi_{1}^{n+1}}{\partial t} \psi_{1} d x d y=\partial_{\phi} F\left(\left(\phi_{1}^{n+1}, \phi_{2}^{n}\right), c\left(\phi^{n}\right)\right)\left(\psi_{1}, 0\right), \quad \forall \psi_{1} \in H^{1}(\Omega) \\
\int_{\Omega} \frac{\partial \phi_{2}^{n+1}}{\partial t} \psi_{2} d x d y=\partial_{\phi} F\left(\left(\phi_{1}^{n}, \phi_{2}^{n+1}\right), c\left(\phi^{n}\right)\right)\left(0, \psi_{2}\right), \quad \forall \psi_{2} \in H^{1}(\Omega) .
\end{array}
$$

We remark that we can as well define a mixed regularized version of this four-phase model, as in section 2.3, by adding to the functional (22) the terms (compare with (15))

$$
\sum_{i=1}^{2}\left(\frac{\beta}{4} \int_{\Omega}\left(\left|\nabla \phi_{i}\right|^{2}-1\right)^{2} d x d y+\frac{\alpha}{2} \int_{\Omega}\left|\nabla \phi_{i}\right|^{2} d x d y\right) .
$$

Moreover this multi-phase model can be extended to vector-valued images (see [38]). In addition, keeping the vector $c=\left(c_{i j}\right)$ fixed, with $i, j=0,1$, it is also possible to reformulate the multi-phase model as a convex unconstrained minimization problem, as described in section 2.5.

\section{Application to ACF's endoscopic images}

We describe now the segmentations obtained for seven different ACF's endoscopic images, using the models of section 2 and the software COMSOL Multiphysics@ [12]. One of the advantages of this software is that it allows the possibility of defining a PDE model in its weak form. Besides, its routines can be coupled with MATLABß [35] routines, for instance optimization functions.

In all the experiments we have used 10 and 30 iterations, for the outer and inner loops, respectively, in the numerical scheme. The initial guess is either one circle or a seed of circles. The domain $\Omega$, representing the given medical images (which have different resolutions, ranging from $200 \times 200$ to $710 \times 710$ pixels) is discretized with regular squared linear finite elements with 50,100, 150 or 200 elements per side. In these numerical experiments the values for the regularizing parameters are as follows (unless otherwise stated): $\mu=220$ (coefficient of the length term), $\eta=0$ (coefficient of the area of the region inside the curve), $\varepsilon=.1$ (parameter in the regularization of the Heaviside and Dirac delta functions), $\theta=1$. and $b=.1$ (coefficient and regularization parameter for the penalty term $p$ in (20)), and $a=.01$ (parameter for the numerical realization of the inverse of the norm of the gradient of the level set function, that is, in $1 /|\nabla \phi|$ the denominator is replaced 
by $\left.|\nabla \phi|_{a}=\sqrt{a+|\nabla \phi|^{2}}\right)$. For the other parameters, i.e., the coefficients of the fitting terms and the regularizing parameters $\beta$ and $\alpha$ defined in section 2.3 , we indicate their values for each medical image. We note however, that we have kept the coefficients of the fitting terms almost constants in many experiments. Theoretically, the larger they are, more accurate and fine is the segmentation (see $[17,36]$ ). In the numerical experiments, we have tested all the six models. Unless otherwise stated, and if the used model is not explicitly mentioned in the text, it is understood that it is the vector-valued model (see section 2.4) and the multi-phase model (more precisely, the four-phase model, see section 2.6) reformulated as convex unconstrained minimization problems, as described in section 2.5. Moreover, in the modified and four phase models we have always chosen the red channel of the given image $u_{0}$ as the input image.

The first Figure 1 shows the segmentations obtained with the four-phase model (described in section 2.6), with and without the mixed regularized terms (the signed distance and Tikhonov tems, see section 2.3 and (27)). The original image has $200 \times 200$ pixels. We use a squared finite element mesh with 100 finite elements per side, and for the initial guess $\phi^{0}=\left(\phi_{1}^{0}, \phi_{2}^{0}\right)$ we choose seeds of circles (depicted in Figure 2, top right, where the white and red circles represent the zero level sets of $\phi_{1}^{0}$ and $\phi_{2}^{0}$, respectively). In the Figure 1, the fitting parameters $\lambda_{i j}$, for $i, j=0,1$ are all equal to 50 and for the regularized case $\beta=10^{-5}$ and $\alpha=10^{-9}$.

In Figure 2 we apply the four-phase regularized model to the same medical image displayed in Figure 1 and we also kept the same values, with respect to the fitting parameters, and the initial guess used in Figure 1. The intention here is to show the influence of the coefficient $\beta$ (in the signed distance function term), in the segmentation results, for a fixed coefficient $\alpha=10^{-9}$ (in the regularized Tikhonov term). We choose $\beta=10^{-6}, 10^{-5}, 10^{-4}$. In addition we have also increased the number of finite elements, from $100^{2}$ to $200^{2}$, while changing $\beta$ from $10^{-5}$ to $10^{-4}$. The same endoscopic image as before, is again used in Figure 3, which presents the segmentations obtained with the four-phase regularized model (with $\alpha=10^{-9}$ and $\beta=10^{-5}$ ). The difference now is that the initial guess is a pair of two circles (see Figure 3 , top left). We note that the separated and overlapped segmentations are different from those shown in Figures 1-2, obtained with a seed of circles as initial zero level set guess. 

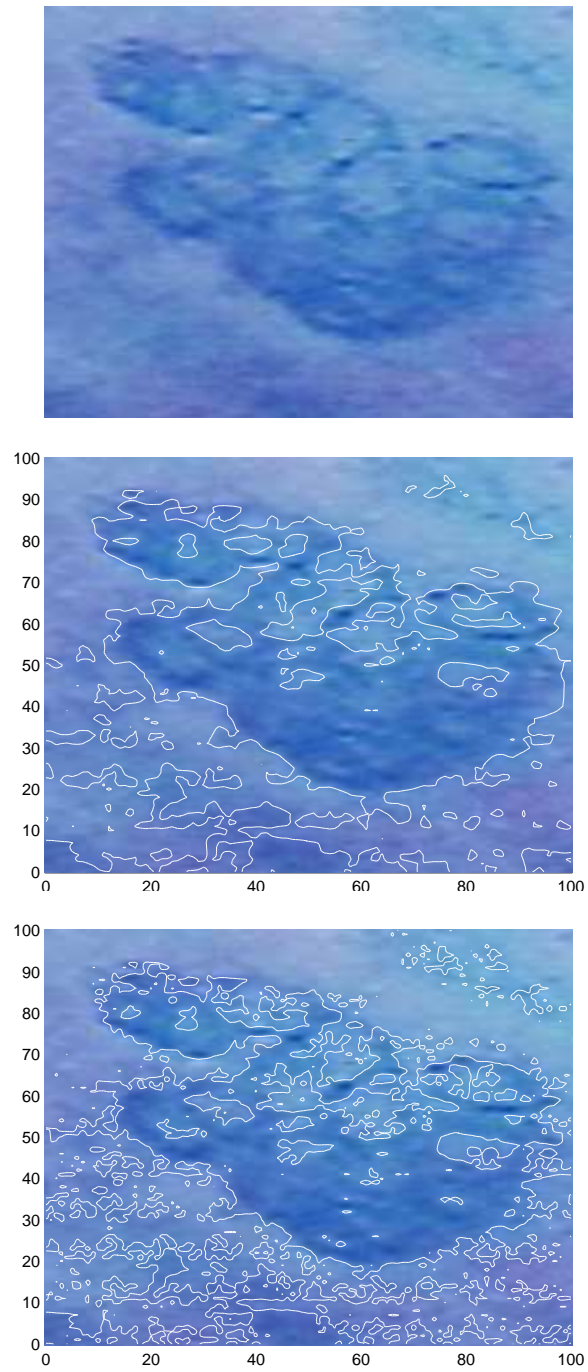
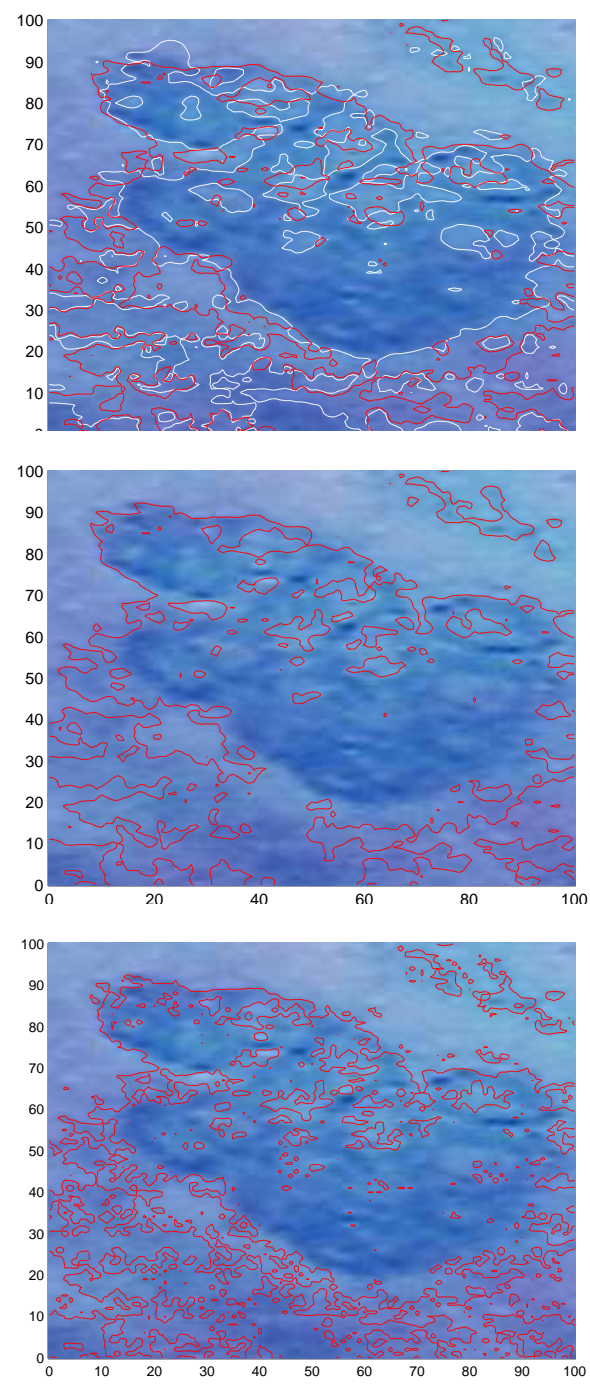

Figure 1. Medical image with $200 \times 200$ pixels (top left) and four-phase segmentation (red and white level set contours) with $100 \times 100$ finite elements: the two separated segmentations with mixed regularization for $\alpha=10^{-9}, \beta=10^{-5}$ (second row) and without regularization (third row) and the overlapped segmentations for the regularized case (top right).

The Figure 4 indicates the segmentation results, still for the same medical image of Figure 1, but with the other models described in the paper. The top right segmentation results correspond to the regularized RGB vector-valued model (the initial guess is a circle and the coefficients of the fitting parameters are $\lambda_{1}^{ \pm}=50, \lambda_{2}^{ \pm}=10$ and $\lambda_{3}^{ \pm}=10$, for the red, green and blue channels, 

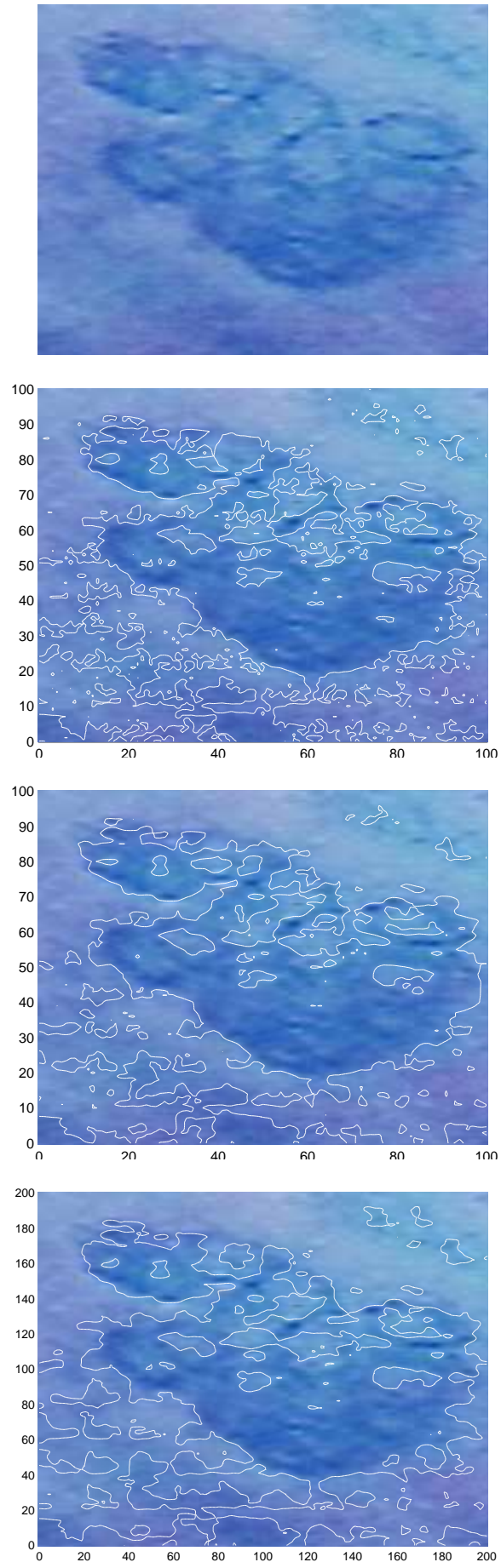
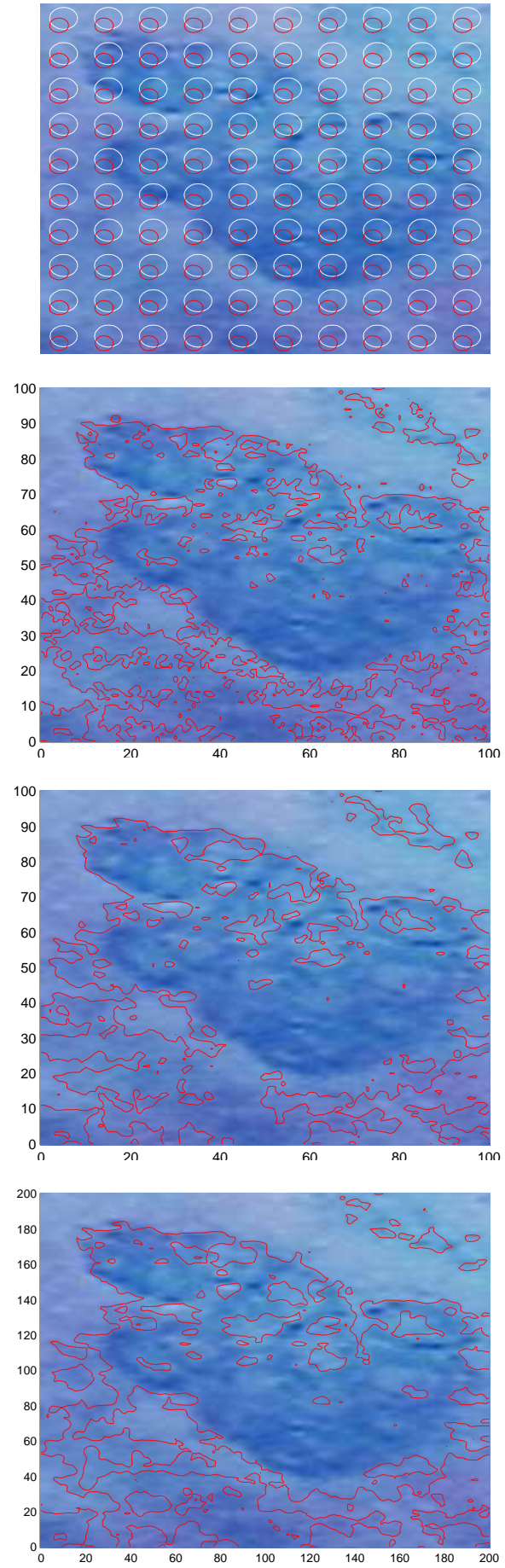

Figure 2. Medical image with $200 \times 200$ pixels (top left) and three four-phase regularized segmentations (red and white level set contours) with $\alpha=10^{-9}$ : initial condition (top right), $\beta=$ $10^{-6}$ and $100 \times 100$ finite elements (second row), $\beta=10^{-5}$ and $100 \times 100$ finite elements (third row) and $\beta=10^{-4}$ and $200 \times 200$ finite elements (fourth row). 

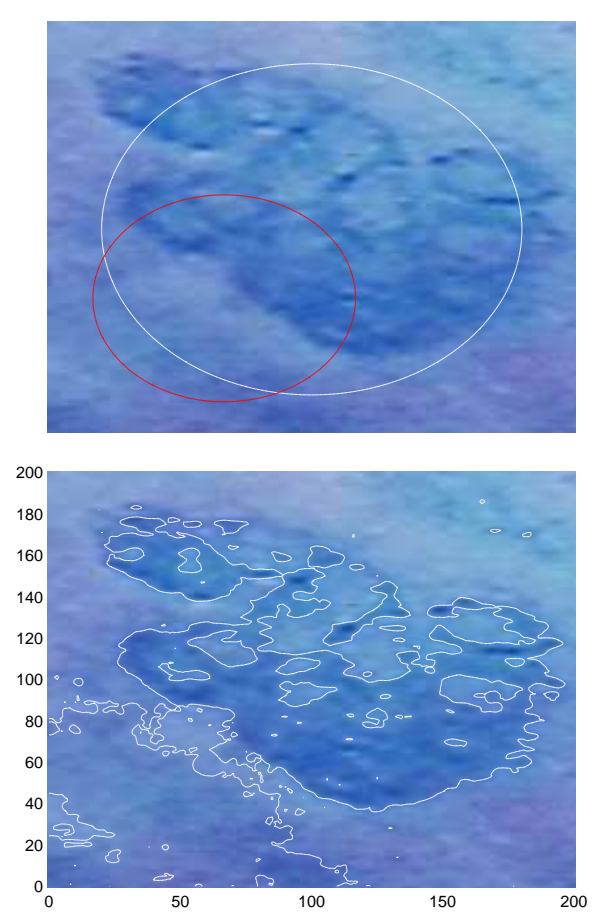
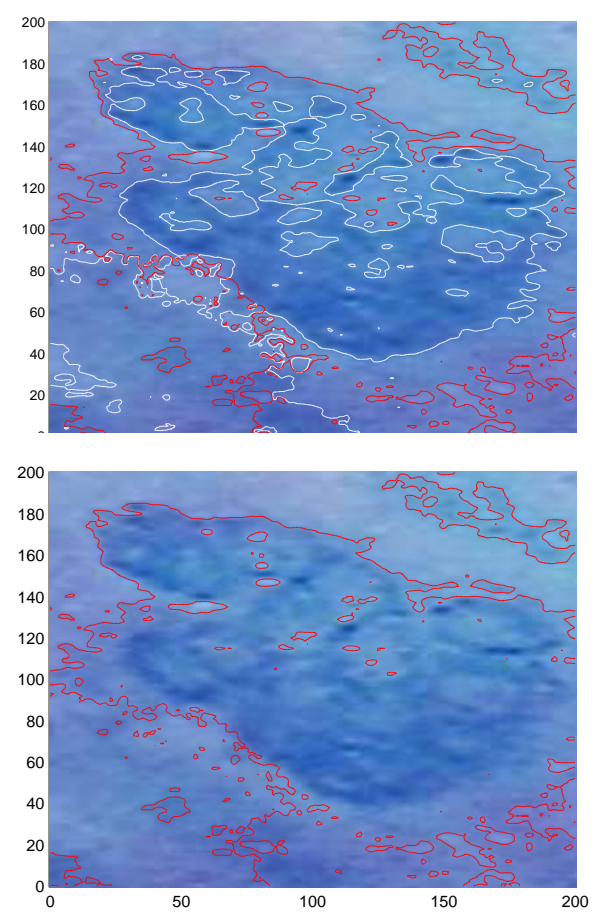

FiguRE 3. Medical image $(200 \times 200$ pixels $)$ with the initial condition superimposed (top left) and one four-phase regularized segmentation (red and white level set contours): overlapped segmentations (top right), separated segmentations with regularization parameters $\alpha=10^{-9}, \beta=10^{-5}$, with $200 \times 200$ finite elements (bottom).

respectively), with the particularity that the regularizing parameters are updated in the outer loop. Their initial values are $\alpha=10$ and $\beta=10^{-15}$ and updated to $\alpha=\alpha \times 10^{-1}$ and $\beta=\beta \times 10$ in each outer iteration (hence the final values are $\alpha=10^{-9}$ and $\beta=10^{-5}$ ). In the middle row of Figure 4 , the two segmentations with $50^{2}$ and $200^{2}$ finite elements are achieved with the modified model, see sections 2.1 and 2.2 (the initial guess is a circle, the coefficients in the fitting terms are all equal to 30 , and the parameter $\varepsilon=8$.). But for the image on the right of the middle row, the modified model is reformulated as a convex unconstrained optimization problem. Finally, the bottom row in Figure 4 shows two segmentations, again with $50^{2}$ and $200^{2}$ finite elements, performed with the vector-valued model for three channels: one is related to the intensity of the RGB image and is the red channel, 

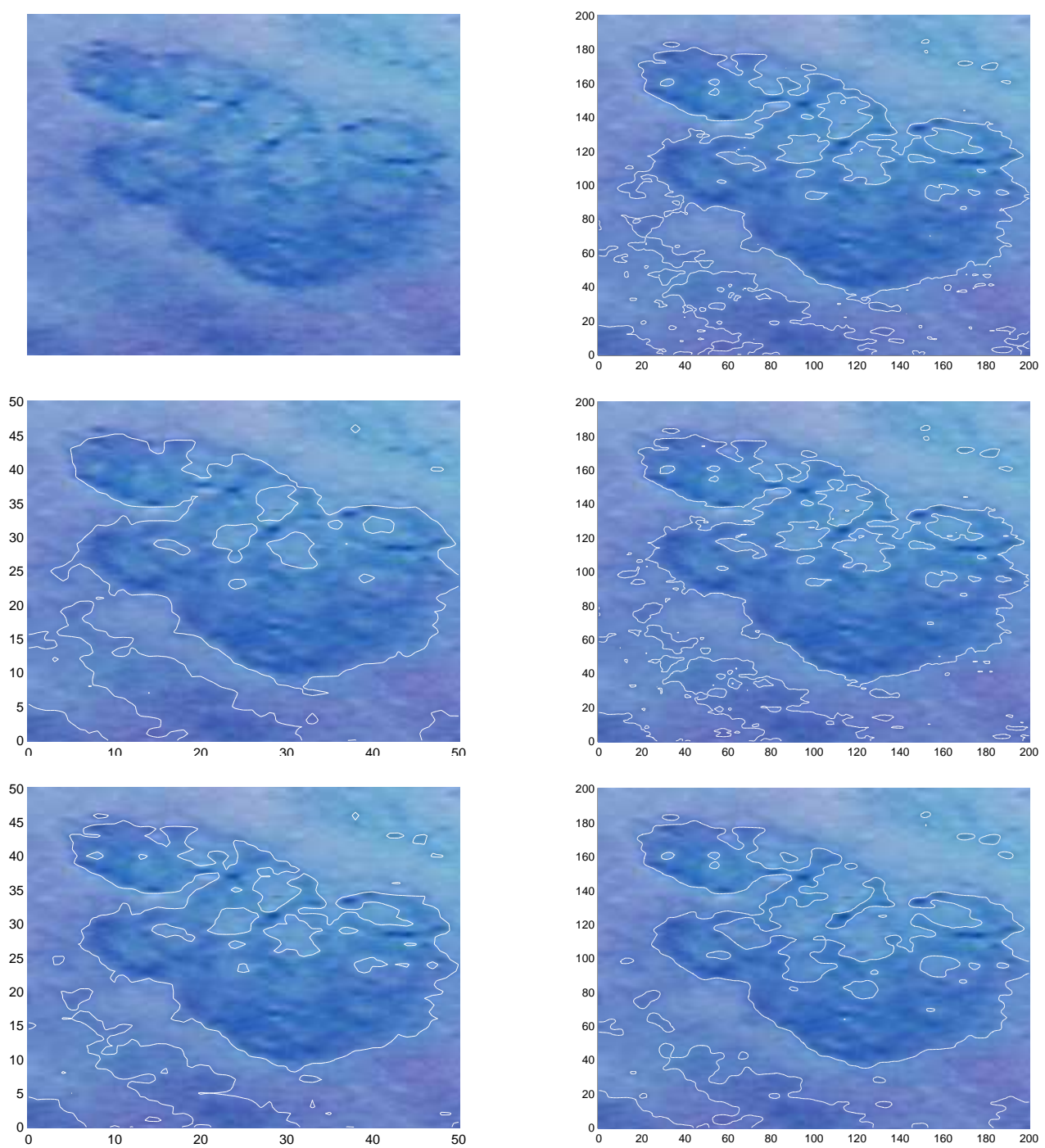

FiguRE 4. Medical image with $200 \times 200$ pixels (top left) and four different segmentations (white contours): regularized RGB vector valued model with $200 \times 200$ finite elements (top right), modified model (second row, left and right) and colored-textured vector valued model (third row, left and right), for $50 \times 50,200 \times$ 200 finite elements and different values for the parameters of the fitting terms.

while the other two channels are linked to the texture of the endoscopic image $u_{0}$, see section 2.4. These are the curvature of $u_{0}\left(\operatorname{curv}\left(u_{0}\right)=\operatorname{div} \frac{\nabla u_{0}}{\left|\nabla u_{0}\right|}\right)$ 
and the orientation of $u_{0}$ (orient $\left(u_{0}\right)=\tan ^{-1}\left(\frac{u_{0, y}}{u_{0, x}}\right)$ ). For the bottom left segmentation, the coefficients in the fitting terms are all equal to 10, while in the bottom right segmentation the fitting coefficients with respect to the red channel are equal to 2 and the others equal to 10. Moreover, for both cases the initial zero level set function is a circle and the parameter $\varepsilon=.01$.
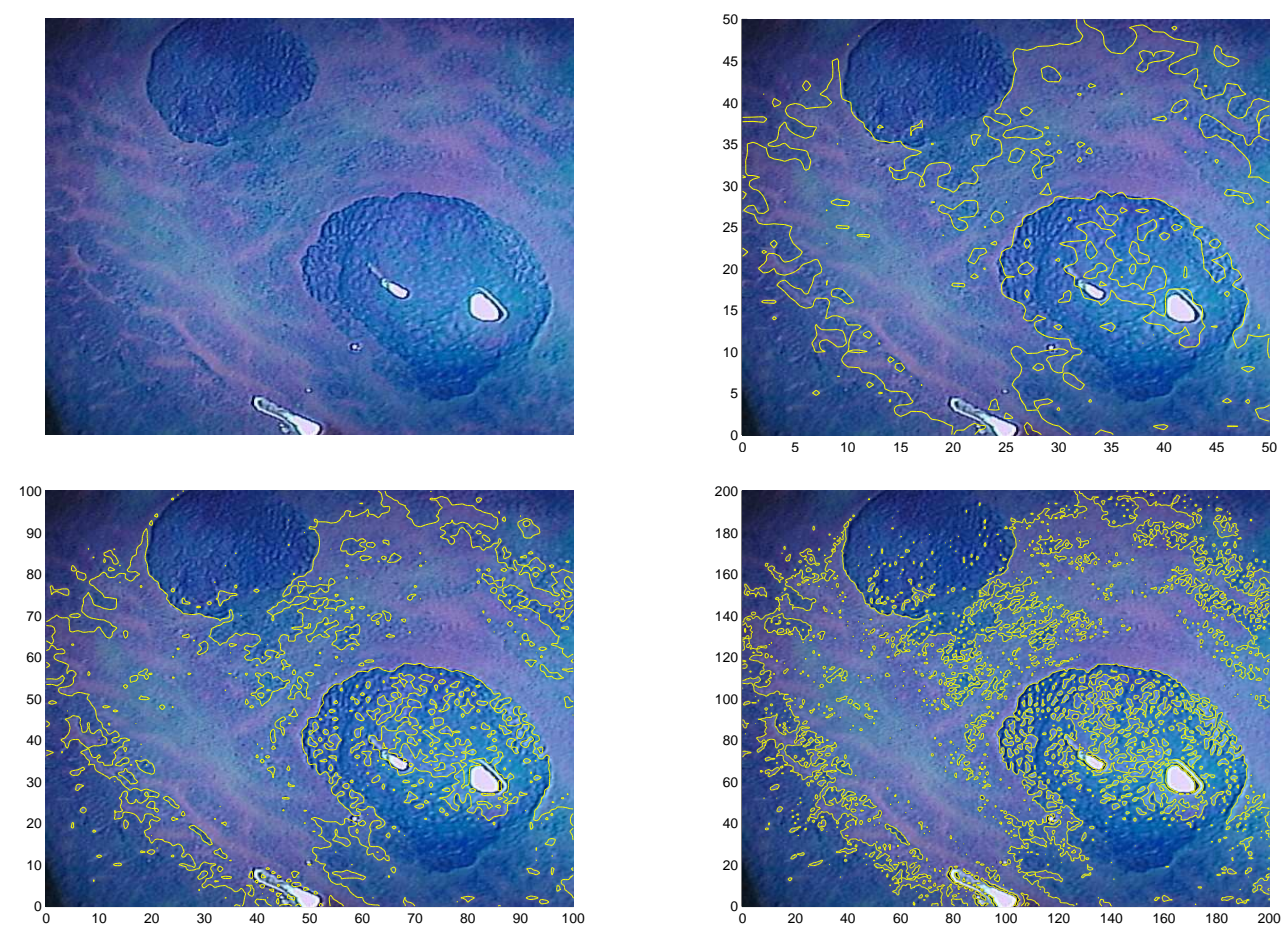

Figure 5. Medical image with $500 \times 500$ pixels (top left) and three segmentations (yellow contours) with the RGB vector valued model: for $50 \times 50,100 \times 100$ and $200 \times 200$ finite elements (top right, bottom right and left).

The Figure 5 exhibits a different ACF endoscopic image with $500 \times 500$ pixels and evidences the influence of the finite element mesh refinement, for the vector-valued model (without signed distance and Tikhonov regularization) with three channels, more exactly the RGB colored image. The initial zero level set function is a seed of circles, and the values of the fitting parameters are $\lambda_{1}^{ \pm}=10, \lambda_{2}^{ \pm}=.3$ and $\lambda_{3}^{ \pm}=.3$, for the red, green and blue channels, respectively. For this medical image, we also show in Figure 6 the segmentations obtained with the modified model, reformulated as a convex unconstrained optimization problem and without regularization (see sections 
2.2 and 2.5), the two separated segmentations resulting from the four-phase regularized model, for $\alpha=10^{-9}$ and $\beta=10^{-5}$, and for the no regularized case. For the modified model, the coefficients in the fitting terms are all equal to 30 and the initial zero level set function is a circle, while for the other model the initial guess is a seed of circles and the former coefficients are all equal to 50.
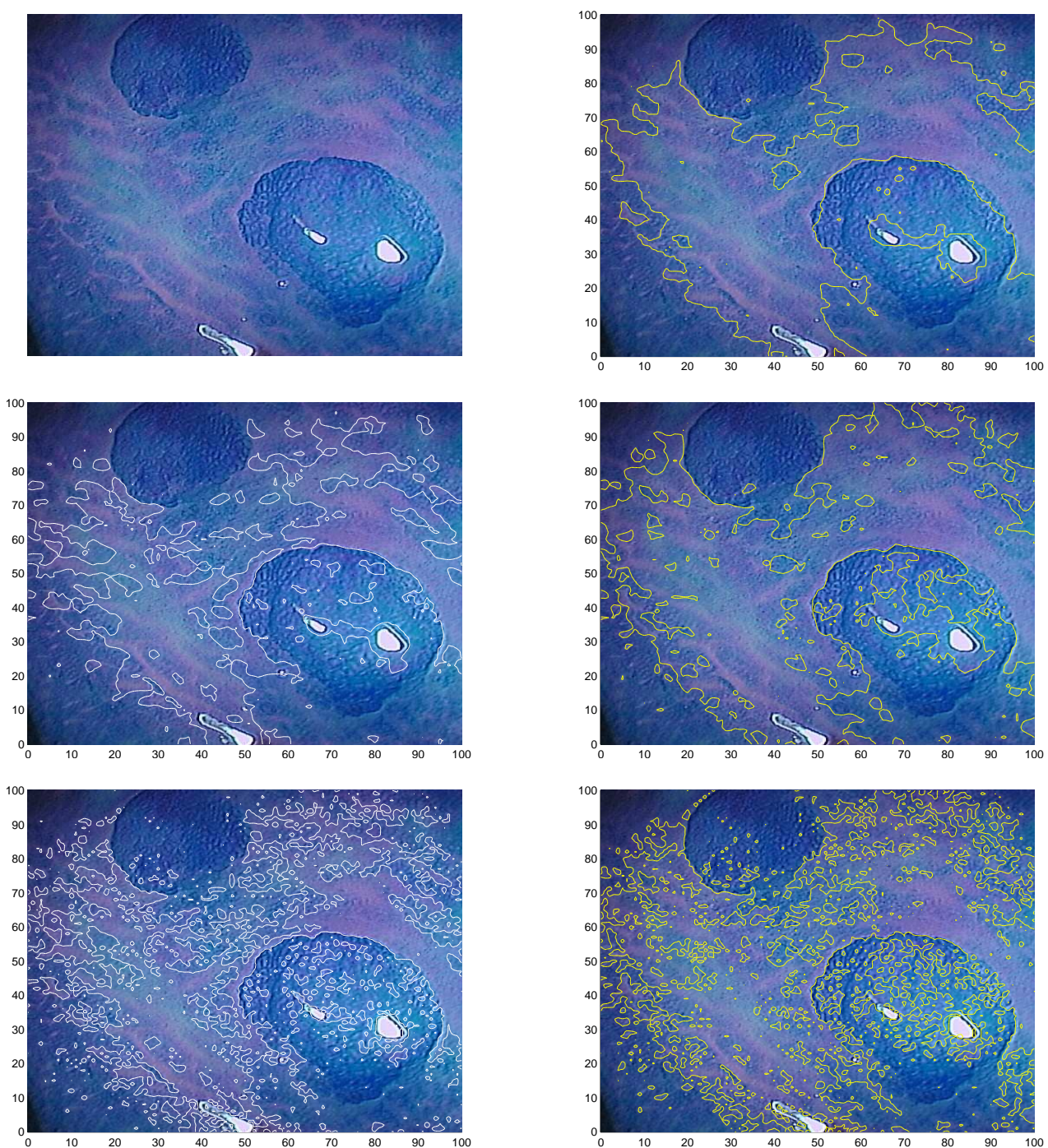

Figure 6. Medical image (top left, with $500 \times 500$ pixels) and five segmentations (yellow and white contours) for $100 \times 100$ finite elements: the modified model (top right), and the four-phase model with regularization for $\alpha=10^{-9}, \beta=10^{-5}$ (second row) and without regularization (third row). 
In Figure 7 we have one more endoscopic image with several ACF and segmentations with three models. The modified model, reformulated as a convex unconstrained optimization problem, where the coefficients in the fitting terms all equal to 30 and the initial guess is a circle. The RGB vectorvalued model with the coefficients of the fitting parameters equal to $\lambda_{1}^{ \pm}=10$, $\lambda_{2}^{ \pm}=.3$ and $\lambda_{3}^{ \pm}=.3$ and the initial guess is a seed of circles. And finally the four-phase regularized model, with the coefficients of the fitting parameters all equal to 50, the initial guess is a pair of seeds of circles and the regularizing parameters $\alpha=10^{-9}, \beta=10^{-5}$ for the third row and $\alpha=10^{-10}, \beta=10^{-6}$ for the fourth row.

The Figure 8 presents three diverse endoscopic images, showing three different kinds of ACF . The corresponding segmentations are achieved with the RGB vector-valued model without regularization, where the coefficients of the fitting parameters are equal to $\lambda_{1}^{ \pm}=50, \lambda_{2}^{ \pm}=10$ and $\lambda_{3}^{ \pm}=10$, for the red, green and blue channels and with a circle as initial zero level set function.

The Figure 9 displays a distinct ACF endoscopic image for which we have tested the modified model, reformulated as a convex unconstrained minimization problem (the coefficients of the fitting terms all equal to 30 and a circle as initial guess), the RGB vector-valued model without regularization (with the coefficients of the fitting terms in the red channel equal to 10 and the others equal to .3, and a seed of circles as initial guess) and the coloredtextured model with regularization (the coefficients of the fitting terms in the red channel equal to 5 , those of the texture fitting terms (the curvature and the orientation of the given image) are equal to $50, \alpha=10^{-9}, \beta=10^{-5}$ and the initial guess is a circle), and also the four-phase regularized model (with $\alpha=10^{-9}, \beta=10^{-5}$, the coefficients of the fitting terms all equal to 50, and a seed of circles as initial guess).

We remark that the white spots, which appear in some of these endoscopic images, correspond to the mucus inside the colon (sometimes it is not possible to avoid them during the endoscopic exam).

\section{Conclusions and future work}

In this paper we have applied different active contour without edges models to segment distinct ACF endoscopic images. All the results were achieved

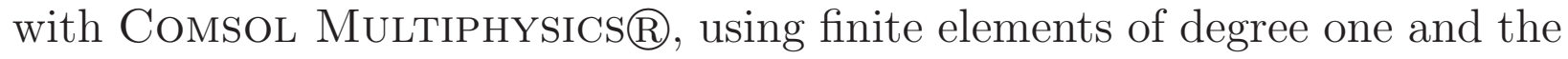
numerical implicit scheme described on page 8 , with the stationary linear 

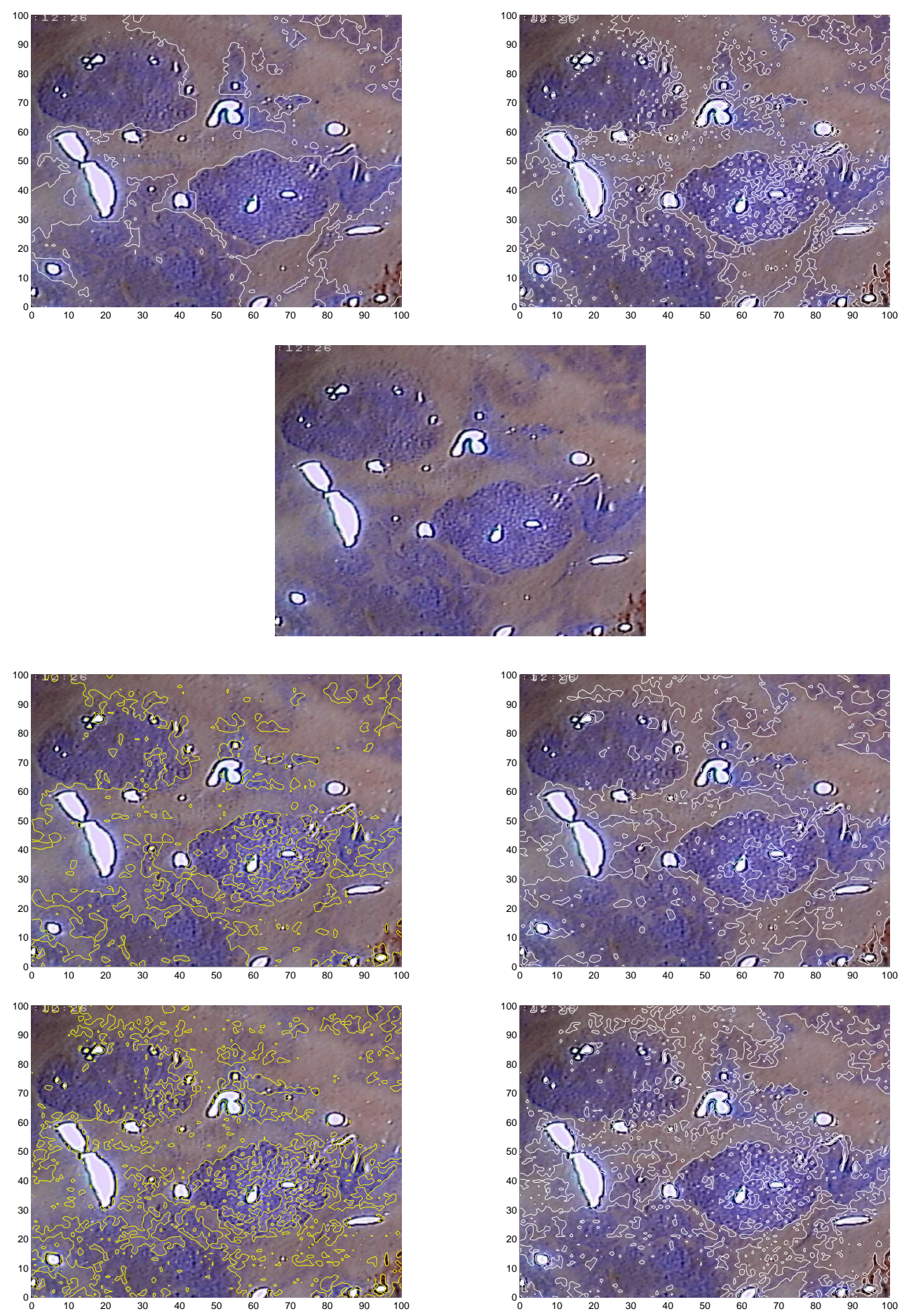

Figure 7. Medical image with $620 \times 620$ pixels (middle row) and five segmentations (white and yellow contours). The modified model (top left), the RGB vector valued model (top right), and the four-phase model with regularization: $\alpha=10^{-9}, \beta=10^{-5}$ (third row) and $\alpha=10^{-10}, \beta=10^{-6}$ (fourth row) (initial guess seeds of circles). 

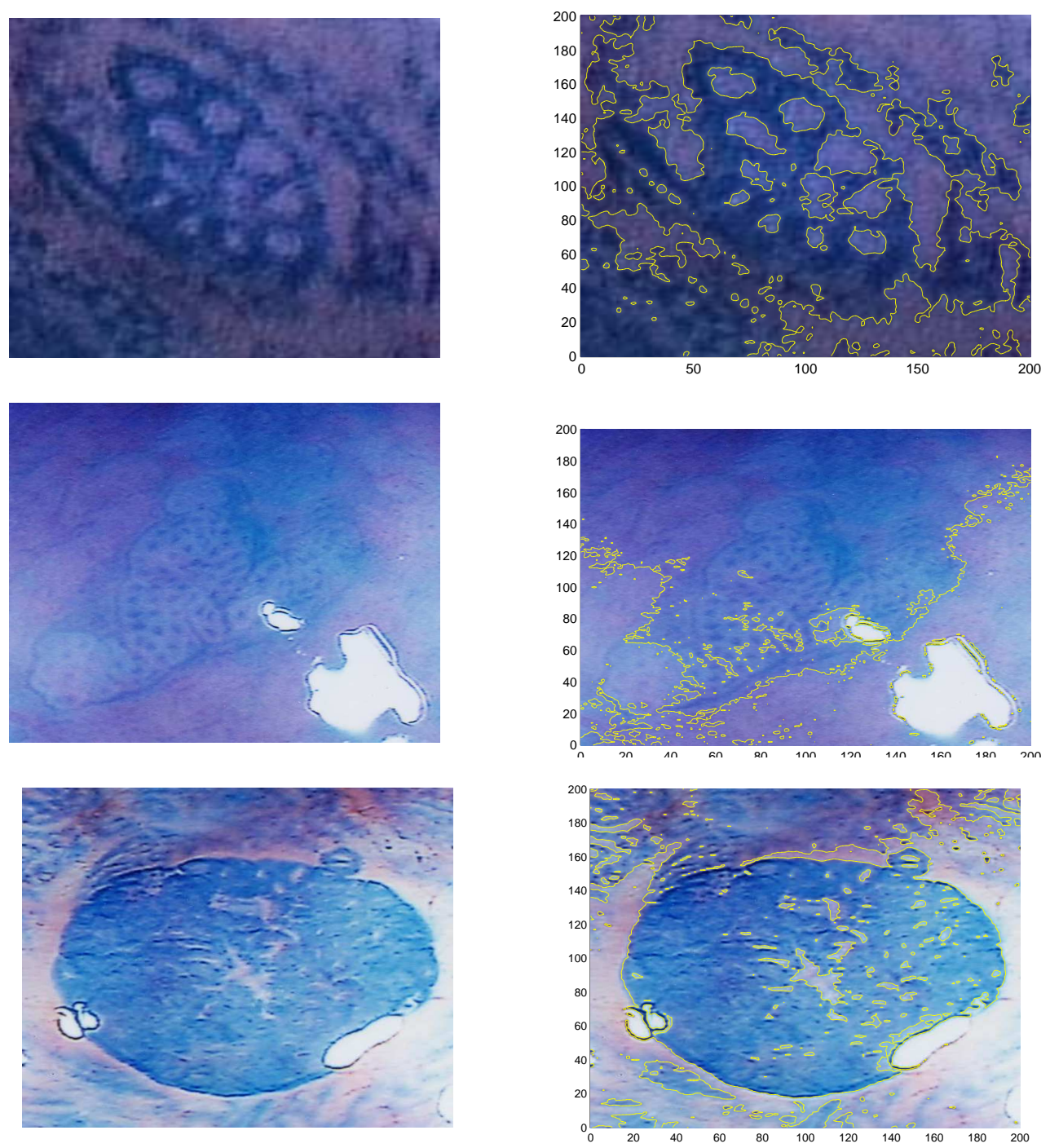

FiguRE 8. Different medical images (left column, with, from top to bottom, $200 \times 200,710 \times 710$ and $560 \times 560$ pixels $)$ and correspondent segmentations (right column, yellow contours) obtained with the RGB vector valued model and $200 \times 200$ finite elements.

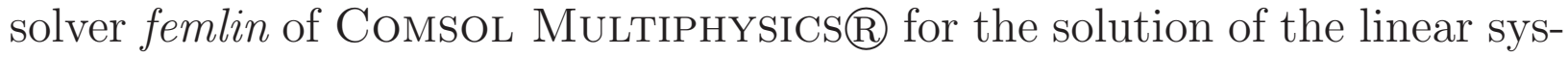
tem (9). The time dependent solver femtime of Comsol Multiphysics $\mathbb{B}$ was not utilized for the solution of (6), since it has been proven a far too slow process for these endoscopic images, and this was one of the reasons why we have developed the algorithm explained on page 8 . 

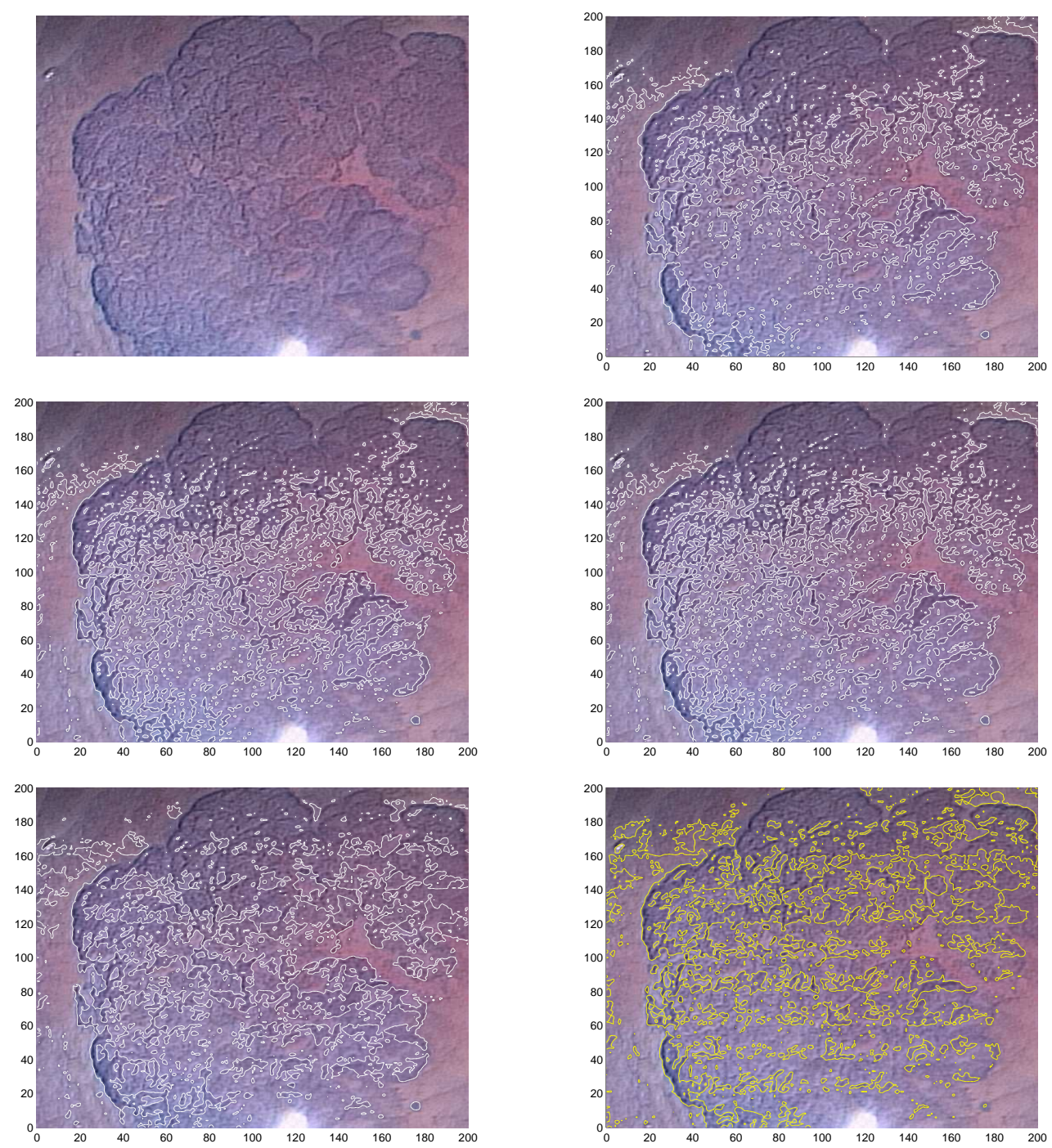

FiguRE 9. Medical image with $500 \times 500$ pixels (top left) and five segmentations (white and yellow contours) with $200 \times 200$ finite elements: modified model (top right) and RGB vector valued model (middle left), colored-textured regularized model with $\alpha=$ $10^{-9}, \beta=10^{-5}$ (middle right) and the two separated level set functions of a four phase regularized segmentation with $\alpha=10^{-9}$, $\beta=10^{-5}$ (bottom left and right).

It is worth mentioning that the coefficients $\alpha$ and $\beta$ (in the Tikhonov and signed distance function regularizing terms, defined in section 2.3), undoubtedly improve the quality of the segmentation, for all the models, and in special for the four-phase model. In the latter case, they are essential to 
have a good understanding of the segmentation contours, which would be very difficult without any regularization at all. However, a good choice of the values $\alpha$ and $\beta$ should be made, to avoid over-smoothed curves, a coarsen segmentation and the risk of loosing some important details.

It should also be stressed that the models reformulated as convex unconstrained optimization problems (see section 2.5), converge remarkably faster than when they are not reformulated in this way.

Regarding the modified model, introduced on section 2.2 (which is essentially the model of [11] but with a varying length term's coefficient) it has given very accurate segmentations for the case of large details.

In general, for all these endoscopic images, a refinement of the finite element mesh leads to a best and fine segmentation.

As we have remarked before, we always employed the extra time variable in the numerical realization. But this could be avoid, as pointed out on section 2.1. Actually, for the medical image depicted on Figure 1, we have made a test, where we have linked the Comsol Multiphysics@ code with

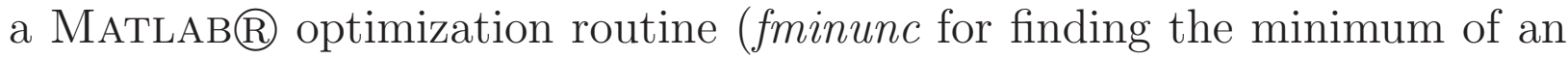
unconstrained multivariable function). However, the execution process was very slow, even for a coarse finite element mesh with $20 \times 20$ finite elements (the algorithm chosen was a large-scale trust-region Netwon method).

We also remark that, for each medical image, of section 3, we did not use the complete endoscopic image, but only a selected region of it, containing the $\mathrm{ACF}$, in order to reduce the size of the problem and speed up the process.

In the future we intend to continue the research on ACF endoscopic image segmentation, with special emphasis on the following issues: the development of a fast and appropriate optimization algorithm (which does not require the introduction of the extra time variable mentioned above), the possibility of exploring parallel computation in order to analyze the complete medical image and not just a small region of it, the incorporation of specific features of the colonic crypts in the PDE-based segmentation models, and finally the image processing of video endoscopic images. In addition, we plan to apply a high-order level set method, based on the generalized MumfordShah functional (cf. [2]), and also a fast marching method (cf. [32, 39]), for segmenting these endoscopic images. Finally, it should not be forgotten, that an ideal tool, very useful to assist doctors, would be the conception of a fast and computerized algorithm able to segment, in real time, the ACF captured 
in vivo by endoscopy. The goal is to pursue the future research along these directions.

\section{References}

[1] D. G. Adler, C. J. Gostout, D. Sorbi, et al. Endoscopic identification and quantification of the aberrant crypt in the human colon. Gastrointestinal Endoscopy, 56:657-662, 2002.

[2] C. Bajaj, G. Xu, and Q. Zhang. A higher-order level-set method with applications to smooth surface constructions. Technical report, University of Texas, Austin, Texas, ICES, TR-06-08, 2006.

[3] B. Berkels, A. Rätz, M. Rumpf, and A. Voigt. Extracting grain boundaries and macroscopic deformations from images on atomic scale. Journal of Scientific Computing, to appear, 2008.

[4] R. P. Bird and C. K. Good. The significance of aberrant crypt foci in understanding the pathogenesis of colon cancer. Toxicology Letters, 112-113:395-402, 2000.

[5] R. P. Bird. Observation and quantification of aberrant crypts in the murine mucosa treated with a colon carcinogen: preliminary findings. Cancer Letters, 37:147-151, 1987.

[6] R. P. Bird. Role of aberrant crypt foci in understanding the pathogenesis of colon cancer. Cancer Letters, 93:55-71, 1995.

[7] V. Caselles, F. Catté, T. Coll, and F. Dibos. A geometric model for active contour in image processing. Numer. Math., 66:1-31, 1993.

[8] T. F. Chan, S. Esedoglu, and M. Nikolova. Algorithms for finding global minimizers of image segmentation and denoising models. SIAM J. Appl. Math., 66(5):1632-1648 (electronic), 2006.

[9] T. F. Chan, B. Y. Sandberg, and L. A. Vese. Active contours without edges for vector-valued images. Journal of Visual Communication and Image Representation, 11(2):130-141, 2000.

[10] T. F. Chan and J. Shen. Image processing and analysis. Society for Industrial and Applied Mathematics (SIAM), Philadelphia, PA, 2005. Variational, PDE, wavelet, and stochastic methods.

[11] T. F. Chan and L. A. Vese. Active contours without edges. IEEE Trans. Image Processing, 10(2):266-277, 2001.

[12] Comsol Multiphysicsß. http://www.comsol.com/.

[13] A. L. Cunha. A fully Eulerian method for shape optimization, with applications to NavierStokes flows. PhD Thesis. Department of Civil and Environmental Engineering, Carnegie Mellon University, Pittsburgh, 2004.

[14] J. P. Silva Cunha, M. Coimbra, P. Campos, and J. M. Soares. Automated topographic segmentation and transit time estimation in endoscopic capsule exams. IEEE Transactions on Medical Imaging, 27(1):19-27, 2008.

[15] D. Drasdo and M. Loeffer. Individual-based models to growth and folding in one-layered tissues: intestinal crypts and early development. Nonlinear Analysis, 47:245-256, 2001.

[16] B. Engquist, A.-K. Tornberg, and R. Tsai. Discretization of Dirac delta functions in level set methods. J. Comput. Phys., 207(1):28-51, 2005.

[17] S. Esedoḡlu and R. Tsai. Threshold dynamics for the piecewise constant Mumford-Shah functional. J. Comput. Phys., 211(1):367-384, 2006.

[18] J. Ferlay, P. Autier, M. Boniol, M. Heanue, M. Colombet, and P. Boyle. Estimates of the cancer incidence and mortality in Europe in 2006. Annals of Oncology, 18:581-592, 2007.

[19] D. R. Halm and S. T. Halm. Secretagogue response of goblet cells and columnar cells in human colonic crypts. Am. J. Physiol. Cell Physiol, 278:212-233, 2000. 
[20] D. Hurlstone et al. Rectal aberrant crypt foci identified using high-magnification-chromoscopic colonoscopy: biomarkers for flat and depressed neoplasia. American Journal of Gastroenterology, pages 1283-1289, 2005.

[21] A. Jemal, R. Siegel, E. Ward, et al. Cancer statistics, 2008. CA: A Cancer Journal for Clinicians, 58:71-96, 2008.

[22] M. D. Johnston, C. M. Edwards, W. F. Bodmer, P. K. Maini, and S. J. Chapman. Mathematical modeling of cell population dynamics in the colonic crypt in colorectal cancer. PNAS, 104(10):4008-4013, 2007.

[23] M. Kass, A. Witkin, and D. Terzopoulos. Snakes: Active contour models. Int. J. Comput. Vis., 1:321-331, 1988.

[24] E. A. Mclellan and R. P. Bird. Aberrant crypts: potential preneoplastic lesions in the murine colon. Cancer Research, 48:6187-6192, 1998.

[25] F. Michor, Y. Iwasa, C. Lengauer, and M. A. Nowak. Dynamics of colorectal cancer. Seminars in Cancer Biology, 15:484-494, 2005.

[26] D. Mumford and J. Shah. Optimal approximations by piecewise smooth functions and associated variational problems. Comm. Pure Appl. Math., 42(5):577-685, 1989.

[27] S. Osher and R. Fedkiw. Level set methods and dynamic implicit surfaces, volume 153 of Applied Mathematical Sciences. Springer-Verlag, New York, 2003.

[28] S. Osher and J. A. Sethian. Fronts propagating with curvature-dependent speed: algorithms based on Hamilton-Jacobi formulations. J. Comput. Phys., 79(1):12-49, 1988.

[29] J. Pitt-Francis et al. Multimodal imaging techniques for the extraction of detailed geometrical and physiological information for use in multi-scale models of colorectal cancer and treatment of individual patients. Computational and Mathematical Methods in Medicine, 7(2-3):177-188, 2006.

[30] X. Qi, Y. Pan, Jr. M. V. Sivak, K. Olowe, and A. M. Rollins. Investigation of computer-aided colonic crypt pattern analysis. In Proceedings of SPIE, Endoscopic Microscopy II, volume 6432, 2007.

[31] L. Roncucci, A. Medline, and W. R. Bruce. Classification of aberrant crypt foci and microadenomas in human colon. Cancer Epidemiology, Biomarkers $\&$ Prevention, 1:57-60, 1991.

[32] J. A. Sethian. A fast marching level set method for monotonically adavancing fronts. Proc. Natl. Acad. Sci. USA, 93:1591-1595, 1996.

[33] J. A. Sethian. Level set methods and fast marching methods, volume 3 of Cambridge Monographs on Applied and Computational Mathematics. Cambridge University Press, Cambridge, second edition, 1999.

[34] T. Takayama, S. Katsuki, Y. Takahashi, et al. Aberrant crypt foci of the colon as precursors of adenoma and cancer. The New England Journal of Medicine, 339:1277-1284, 1998.

[35] The Mathworks, InC. http://www.matlab.com.

[36] R. Tsai and S. Osher. Total variation and level set methods in image science. Acta Numer., 14:509-573, 2005.

[37] I. M. M. van Leeuwen, H. M. Byrne, O. E. Jensen, and J. R. King. Crypt dynamics and colorectal cancer: advances in mathematical modelling. Cell Prolif., 39:157-181, 2006.

[38] L. Vese and T. F. Chan. A multiphase level set framework for image segmentation using the Mumford and Shah model. International Journal of Computer Vision, 50(3):271-293, 2002.

[39] Z. Yu and C. Bajaj. Automatic ultrastructure segmentation of reconstructed cryoem maps of icosahedral viruses. In IEEE Transactions on Image Processing: Special Issue on Molecular and Cellular Bioimaging, volume 14, pages 1324-1337, 2005. 
I. N. Figueiredo

CMUC, Department of Mathematics, University of Coimbra, 3001-454 Coimbra, Portugal

E-mail address: isabelf@mat.uc.pt

P. N. Figueiredo

Faculty of Medicine, University of Coimbra and Department of Gastroenterology, University Hospital of Coimbra, 3000-075 Coimbra, Portugal

E-mail address: pedro.n.figueiredo@clix.pt

G. STADLER

Institute for Computational Engineering and Sciences (ICES), University of TeXas at Austin, 1 University Station C0200, Austin, TX 78712, USA.

E-mail address: georgst@ices.utexas.edu

O. Ghattas

Institute for Computational Engineering and Sciences (ICES), University of Texas at Austin, 1 University Station C0200, Austin, TX 78712, USA.

E-mail address: omar@ices.utexas.edu

A. Araújo

CMuC, Department of Mathematics, University of Coimbra, 3001-454 Coimbra, Portugal

E-mail address: alma@mat.uc.pt 